\title{
Introducing uncertainty analysis of nucleation and crystal growth models in Process Analytical Technology (PAT) system design of crystallization processes
}

\author{
Abdul Samad, Noor Asma Fazli Bin; Sin, Gürkan; Gernaey, Krist; Gani, Rafiqul
}

Published in:

European Journal of Pharmaceutics and Biopharmaceutics

Link to article, DOI:

10.1016/j.ejpb.2013.05.016

Publication date:

2013

Link back to DTU Orbit

Citation (APA):

Abdul Samad, N. A. F. B., Sin, G., Gernaey, K., \& Gani, R. (2013). Introducing uncertainty analysis of nucleation and crystal growth models in Process Analytical Technology (PAT) system design of crystallization processes. European Journal of Pharmaceutics and Biopharmaceutics, 85(3), 911-929. https://doi.org/10.1016/j.ejpb.2013.05.016

\section{General rights}

Copyright and moral rights for the publications made accessible in the public portal are retained by the authors and/or other copyright owners and it is a condition of accessing publications that users recognise and abide by the legal requirements associated with these rights.

- Users may download and print one copy of any publication from the public portal for the purpose of private study or research.

- You may not further distribute the material or use it for any profit-making activity or commercial gain

- You may freely distribute the URL identifying the publication in the public portal 
Research paper

\title{
Introducing uncertainty analysis of nucleation and crystal growth models in Process Analytical Technology (PAT) system design of crystallization processes
}

\author{
Noor Asma Fazli Abdul Samad ${ }^{a}$, Gürkan Sin ${ }^{\text {a }}$, Krist V. Gernaey ${ }^{\mathrm{b}}$, Rafiqul Gani ${ }^{\mathrm{a}, *}$ \\ ${ }^{a}$ Computer Aided Process-Product Engineering Center (CAPEC), Department of Chemical and Biochemical Engineering, Technical University of Denmark, DK-2800, \\ Kgs. Lyngby, Denmark \\ ${ }^{\mathrm{b}}$ Center for Process Engineering and Technology (PROCESS), Department of Chemical and Biochemical Engineering, Technical University of Denmark, DK-2800, Kgs. Lyngby, Denmark
}

\section{A R T I C L E I N F O}

\section{Article history:}

Received 6 September 2012

Accepted in revised form 31 May 2013

Available online $\mathrm{xxxx}$

\section{Keywords:}

Crystallization

Mathematical model

PAT system design

Uncertainty and sensitivity analysis

Monte Carlo

SRC method

Morris screening

CSD

\begin{abstract}
A B S T R A C T
This paper presents the application of uncertainty and sensitivity analysis as part of a systematic modelbased process monitoring and control (PAT) system design framework for crystallization processes. For the uncertainty analysis, the Monte Carlo procedure is used to propagate input uncertainty, while for sensitivity analysis, global methods including the standardized regression coefficients (SRC) and Morris screening are used to identify the most significant parameters. The potassium dihydrogen phosphate (KDP) crystallization process is used as a case study, both in open-loop and closed-loop operation. In the uncertainty analysis, the impact on the predicted output of uncertain parameters related to the nucleation and the crystal growth model has been investigated for both a one- and two-dimensional crystal size distribution (CSD). The open-loop results show that the input uncertainties lead to significant uncertainties on the CSD, with appearance of a secondary peak due to secondary nucleation for both cases. The sensitivity analysis indicated that the most important parameters affecting the CSDs are nucleation order and growth order constants. In the proposed PAT system design (closed-loop), the target CSD variability was successfully reduced compared to the open-loop case, also when considering uncertainty in nucleation and crystal growth model parameters. The latter forms a strong indication of the robustness of the proposed PAT system design in achieving the target CSD and encourages its transfer to full-scale implementation.
\end{abstract}

(c) 2013 Elsevier B.V. All rights reserved.

\section{Introduction}

Crystallization is often applied in the production of salts and/or active pharmaceutical ingredients (API), and the crystallization step is in general an essential part of the manufacturing process for many chemical-based products. The main specifications of the crystal product are usually given in terms of crystal size, size distribution, shape, and purity. In order to achieve such a set of crystal product specifications consistently, a generic systematic design framework for monitoring and control (PAT) systems has been developed [1]. In the design framework, the necessary problemchemical system specific model(s) can be generated to represent the crystallization process that is studied. The necessary supersaturation set-point and a Process Analytical Technology (PAT) system design can be generated afterward to ensure that the critical process variables are measured and maintained within the design

\footnotetext{
* Corresponding author. Computer Aided Process-Product Engineering Center (CAPEC), Department of Chemical and Biochemical Engineering, Technical University of Denmark, DK-2800 Kgs. Lyngby, Denmark. Tel.: +45 45252882; fax: +45 45932906.

E-mail address: rag@kt.dtu.dk (R. Gani).
}

limits (design space), finally producing a product with the desired specifications.

However, so far in model-based PAT system design, it has been assumed that the exact values of the model parameters are known, for example in the nucleation and crystal growth rate expressions $[1,2]$. These parameters are usually estimated from experimental data, often with considerable measurement errors that also implies a certain error on the estimated parameters. Consequently, there is a degree of uncertainty around the values of nucleation and crystal growth model parameters, which must be taken into account to design a reliable and robust PAT system. In addition, high product quality is usually achieved by implementing model-based optimization approaches, and the benefits to product quality could be lost if uncertainties were not taken into account [3]. In the crystallization process, several approaches have been taken in the past to deal with uncertainties by incorporating robustness in the control of crystallization [4-7]. Nagy et al. proposed a robust online model-based optimization algorithm using distributional batch nonlinear model predictive control (NMPC) which considers the nucleation parameter uncertainties in the optimization problem formulation to determine the robust operating profiles [5]. The 


\section{Nomenclature}

$A_{1}$

$A_{2}$

$a_{i 1}$

$a_{x i}$

$B_{\text {nuc }}$

$b$

$b_{i 1}$

$b_{x i}$

$c$

$c^{\text {sat }}$

$c_{i 1}$

$c_{p}$

$c_{p w}$

$c_{y j}$

$d_{i 1}$

$d_{y j}$

$F_{\text {win }}$

$f_{i j}^{l}$

$f_{i j}^{t}$

$f_{i j}^{0}$

$f_{i j}^{O}$

$f^{n}$

$f_{n 0}$

$G_{x}$

$G_{y}$

$g_{x}$

$g_{y}$

$k$

$k_{b}$

$k_{g x}$

$k_{g y}$

$k_{v}$

$L_{x}$ crystallizer's internal area $\left(\mathrm{cm}^{2}\right)$

crystallizer's external area $\left(\mathrm{cm}^{2}\right)$

polynomial coefficient for saturation concentration

tailor development coefficient

birth rate due to nucleation (number of particles $/ \mathrm{cm}^{3} \mathrm{~s}$ )

nucleation order

polynomial coefficient for saturation concentration

tailor development coefficient

solute concentration (g solute/g solvent)

saturation concentration ( $g$ solute/g solvent)

polynomial coefficient for saturation concentration

heat capacity $\left(\mathrm{J} / \mathrm{g}{ }^{\circ} \mathrm{C}\right)$

water heat capacity $\left(\mathrm{J} / \mathrm{g}{ }^{\circ} \mathrm{C}\right)$

tailor development coefficient

polynomial coefficient for saturation concentration

tailor development coefficient

cooling water flow rate $\left(\mathrm{cm}^{3} / \mathrm{min}\right)$

inlet crystal number flow in the length direction

inlet crystal number flow in the width direction

outlet crystal number flow in the length direction

outlet crystal number flow in the width direction

final population density function (number of particles/ $\mu \mathrm{m})$

initial population density function (number of particles/

$\mu \mathrm{m})$

crystal growth rate in length direction $(\mu \mathrm{m} / \mathrm{s})$

crystal growth rate in width direction $(\mu \mathrm{m} / \mathrm{s})$

growth constant order in length direction

growth constant order in width direction

number of considered variables (factors)

kinetic coefficient for nucleation (number of particles/ $\mathrm{cm}^{3} \mathrm{~min}$ )

kinetic coefficient for crystal growth in length direction $(\mu \mathrm{m} / \mathrm{s})$

kinetic coefficient for crystal growth in width direction $(\mu \mathrm{m} / \mathrm{s})$

crystal shape factor

length of crystal particles $(\mu \mathrm{m})$
$L_{y} \quad$ width of crystal particles $(\mu \mathrm{m})$

$M_{c} \quad$ total crystal mass (g)

$m_{w} \quad$ mass of solvent $(\mathrm{g})$

$N_{c} \quad$ total number of particles (number of particles)

$N_{i} \quad$ number of crystals per unit volume of suspension for class $i$ (number of particles $/ \mathrm{cm}^{3}$ )

$N_{i j} \quad$ number of crystals per unit volume of suspension for class $i$ and $j$ (number of particles $/ \mathrm{cm}^{3}$ )

$p_{x} \quad$ size dependent growth constant for length direction

$p_{y} \quad$ size dependent growth constant for width direction

$R^{2} \quad$ regression of the polynomial model

$S \quad$ supersaturation

$S_{s p} \quad$ supersaturation set-point (g solute/g solvent)

$T \quad$ solution temperature $\left({ }^{\circ} \mathrm{C}\right)$

$T_{e x} \quad$ exterior temperature $\left({ }^{\circ} \mathrm{C}\right)$

$T_{w} \quad$ cooling water temperature $\left({ }^{\circ} \mathrm{C}\right)$

$T_{\text {win }} \quad$ inlet cooling water temperature $\left({ }^{\circ} \mathrm{C}\right)$

$U_{1} \quad$ heat transfer coefficient for internal crystallizer ( $\mathrm{J} /$ ${ }^{\circ} \mathrm{C} \min \mathrm{cm}^{2}$ )

$U_{2} \quad$ heat transfer coefficient for external crystallizer ( $\mathrm{J} /$ ${ }^{\circ} \mathrm{C} \min \mathrm{cm}^{2}$ )

$V \quad$ solution volume $\left(\mathrm{cm}^{3}\right)$

$V_{w} \quad$ cooling water volume $\left(\mathrm{cm}^{3}\right)$

\section{Greek letters}

$\mu_{i} \quad$ mean of distribution function in Morris screening

$\beta_{i} \quad$ standardized regression coefficient

$\sigma_{i} \quad$ standard deviation of distribution function in Morris screening

$\gamma_{x} \quad$ size dependent growth constant for length direction

$\gamma_{y} \quad$ size dependent growth constant for width direction

$\Delta C l_{i} \quad$ extent of $i$ th classes $(\mu \mathrm{m})$

$\Delta C l_{j} \quad$ extent of $j$ th classes $(\mu \mathrm{m})$

$\Delta H_{c} \quad$ heat of crystallization $(\mathrm{J} / \mathrm{g})$

$\rho_{c} \quad$ crystal density $\left(\mathrm{g} / \mathrm{cm}^{3}\right)$

$\rho_{w} \quad$ water density $\left(\mathrm{g} / \mathrm{cm}^{3}\right)$
Monte Carlo simulations were then performed offline by randomly sampling the uncertain parameter space and applying the robust operating profiles. By performing this approach, the variability in the product crystal size distribution (CSD) has been significantly reduced. However, the uncertainties around the crystal growth parameters are not considered in their work and the impact of individual input uncertainty contributions on the output are not quantified. In the work of Saengchan et al., given uncertain kinetic parameters, improvement of batch crystallization control of potassium sulfate has been proposed using model predictive control (MPC) [4].

Furthermore, the impact of parameter uncertainty and control implementation inaccuracies on the performance of the optimal control trajectory are quantified in the work of Ma et al. [8]. These quantitative estimates are then used to decide whether more laboratory experiments are needed to provide more accurate parameter values or to define performance objectives for control loops that implement the optimal control trajectory. As a result, a robust feedback control, for example whether using a simple PID controller or a more advanced controller such as MPC, is needed to deal with uncertainties and to ensure that the desired crystal product is achieved. Before deciding for an appropriate approach to deal with uncertainties in the crystallization process, foremost the impact of such model parameter uncertainties on the predicted system performance needs to be quantified and evaluated. Such an evaluation is useful to find out whether the considered uncertainties may lead to a situation where the target specifications of the crystal product are no longer reached. The latter situation is of course not desirable in a pharmaceutical production process. This requires expansion of model-based methods with formal uncertainty and sensitivity analysis in a comprehensive way.

Uncertainty and sensitivity analysis are usually (and preferably) performed together [9]. Uncertainty analysis is concerned with propagation of the various sources of uncertainty, e.g., input data, stoichiometric parameters, kinetic parameters, etc. to the model outputs, e.g., a performance index. The uncertainty analysis results in probability distributions of model predictions, which are then used to infer the mean, variance, and percentiles of the model predictions. Basically, there are numerous techniques to perform the uncertainty analysis including linear error propagation [10], the Monte Carlo procedure [9,11,12], and fast probability integration (FPI) [13]. In this work, the Monte Carlo procedure is employed for propagation of uncertainty. This is due to the fact that the method is widely accepted as an engineering standard and is computationally efficient as well [12].

The sensitivity analysis, on the other hand, complements uncertainty analysis as it aims at identifying and quantifying the 
individual contributions of the uncertain inputs to the output uncertainty. The sensitivity analysis can be viewed as an analysis of variance, in which the aim is to decompose the output variance with respect to input parameters $[12,14]$. Based on the individual contribution of parameters to the total variance in model predictions, a parameter significance ranking is obtained. This parameter ranking is useful since the efforts can then be focused on reducing the uncertainty in the most influential parameters, rather than spending effort on identifying those parameters that have little or no influence on the model output. In this study, the parameter significance ranking is obtained using two sensitivity analysis methods: (1) linear regression of Monte Carlo outputs, i.e., the standardized regression coefficients (SRC) method [15,16]; and (2) Morris screening [17-19] which produces the mean and standard deviation of the elementary effects of parameters on the outputs.

Identifying the sources of uncertainty is an important issue to be considered before implementing the uncertainty analysis. One of the sources of uncertainty in the system is measurement uncertainty. However, it is a source of uncertainty which can be considered as "known uncertainty" for which there are many methods and tools available in modern control engineering to effectively handle it (such as signal filtering, Kalman filter, extended Kalman filter (EKF), particle filters and robust control theory to name just a few). These tools are easily accessible and presented both in textbooks as well as the open literature [20,21]. In this study, we focus on the kinetic parameters of the crystallization process as a source of uncertainty which we consider as a "known unknown" type of uncertainty which has implications on the probability of successful PAT/process design. This type of uncertainty, to our best knowledge, remains an open problem which we address in this manuscript. Hence, to sum up, in this manuscript, we purposefully focus on parametric uncertainties and present a methodological approach to assess their significance on PAT system design and performance, and suggest a robust PAT design.

In this study, the generic framework for model-based PAT system design of Samad et al. [1] has been expanded with uncertainty and sensitivity analysis tools and methods with the aim to comprehensively test and develop robust and reliable PAT system designs that can cope with the uncertainty. In particular for PAT system design, the objective of this work is to study and analyze the impact of uncertainties in the nucleation and crystal growth parameters on the product-process performances (e.g. CSD) of a crystallization process. The uncertainty and sensitivity analysis is performed in open-loop and closed-loop with two respective aims: in open-loop, the aim is to understand and identify key parameters that drive crystallization performance metrics (product CSD, etc.) and to establish a basis for comparison with the output uncertainties in the closed-loop scenario. In the closed-loop scenario, the aim is to comprehensively test the PAT system design performance in delivering the desired product characteristics under the considered domain of uncertainties. The application of uncertainty and sensitivity analysis is highlighted through a case study of potassium dihydrogen phosphate (KDP) crystallization process, where it will be shown that the effect of the parametric uncertainties on the outputs (product quality) can be minimized by a proposed PAT system design. Moreover, it is also demonstrated that target specifications can be achieved under the considered domain of uncertainties, thus ensuring that the PAT system design is robust.

\section{Expanding the generic framework for model-based PAT system design with uncertainty and sensitivity analysis}

A model-based systematic design framework for monitoring and control (PAT) systems of crystallization processes has been developed earlier [1] and is shown in Fig. 1 (left). Through this framework, it is possible to generate a large number of problemchemical system specific models which can subsequently be used to design a PAT system. In this study, the methods for performing uncertainty and sensitivity analysis adopted from Sin et al. $[9,16]$ have been added as a new feature into the generic framework (see Fig. 1, right) and will be explained in more detail below.

\subsection{Framing of uncertainty and sensitivity analysis (Step 5.1)}

This step deals with the identification, understanding, calculation, and analysis of uncertainties in the model predictions and includes the following sub-steps: (i) identification and characterization of various sources of uncertainties; (ii) Monte Carlo procedure; and (iii) sensitivity analysis.

\subsubsection{Identify sources of uncertainties (Step 5.1.1)}

In this study, we consider uncertainties in the input parameters to the model. To characterize the degree of uncertainty (i.e., the range of uncertainties), the expert review process is used $[9,12]$. In the expert review, the available process expertise and knowledge on the crystallization process are considered in combination with the relevant literature resources to identify uncertain parameters and assign an appropriate range of uncertainty to each parameter, e.g., what is the upper and lower bound of kinetic parameters. In order to structure the expert review process, all the selected model parameters are assumed to follow a uniform probability distribution and three classes of uncertainty are defined $(5 \%, 25 \%$, and $50 \%$ of variability around the mean values [9]). The minimum and maximum values of the uniform distribution can then be calculated as $(100 \%-\%$ variation $) \times$ mean and $(100 \%+\%$ variation $) \times$ mean, respectively. Alternatively, and if available, the lower and upper bound values of the kinetic parameters can be obtained from parameter estimation techniques, e.g., by using the $95 \%$ confidence interval of the estimated model parameters. However, the latter of course requires that experimental data are available.

\subsubsection{Monte Carlo procedure (Step 5.1.2)}

In order to propagate different sources of uncertainties to the model predictions, the Monte Carlo procedure is applied and it involves three sub-steps: (1) sampling of uncertainties; (2) Monte Carlo simulations; and (3) evaluation of output uncertainties.

2.1.2.1. Sampling of uncertainties (Step 5.1.2.1). The input uncertainties specified in the earlier step are sampled using the Latin-Hypercube sampling (LHS) method, a commonly used method [12]. Here, the user has to specify the number of samples. Based on the specified number of samples, a random combination of the uncertain model parameters is then generated and will be used as input to the Monte Carlo simulations. In case, there is a known correlation between the parametric uncertainties, for example on the basis of the results of parameter estimation on available process data, the Iman and Conover method of correlation control can be used to take such correlation into account [22].

2.1.2.2. Monte Carlo simulations (Step 5.1.2.2). The next step in the Monte Carlo procedure is to perform simulations. Here, the mathematical model of the crystallization process is simulated for each set of parameter samples obtained in the previous steps. In this work, both a one- and a two-dimensional mathematical model of KDP crystallization were chosen for Monte Carlo simulations.

2.1.2.3. Evaluation of output uncertainties (Step 5.1.2.3). The results from the Monte Carlo simulations are analyzed in this step by calculating typical statistics such as mean, standard deviation, and relevant percentiles of model output distributions. The uncertainty 


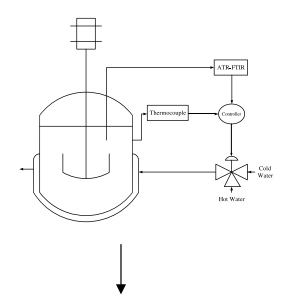

"Crystallization Process"
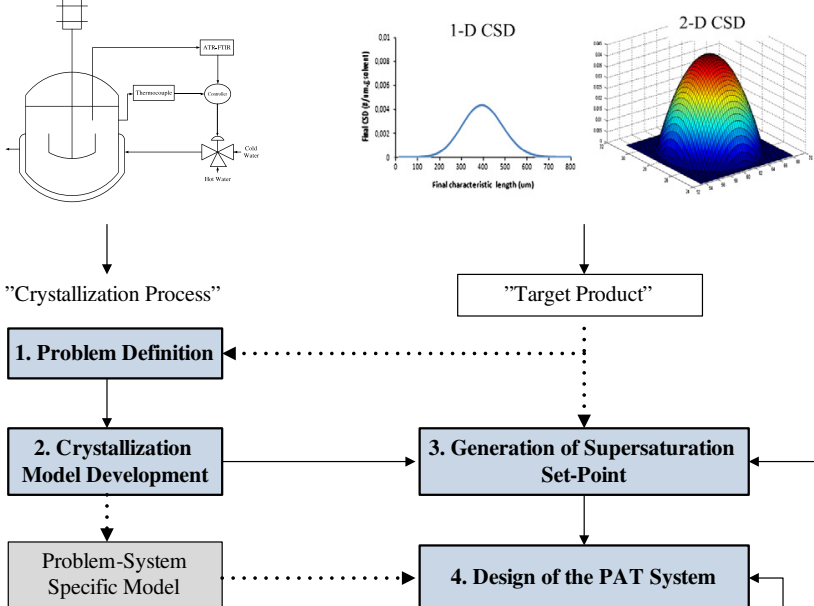

Specific Model

4

Data Handling and Model Identification

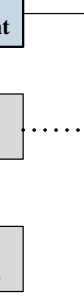

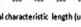

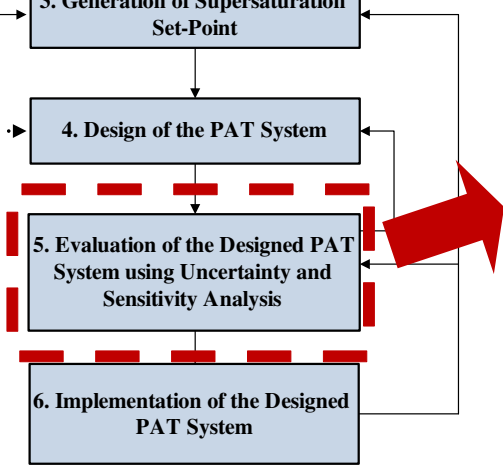

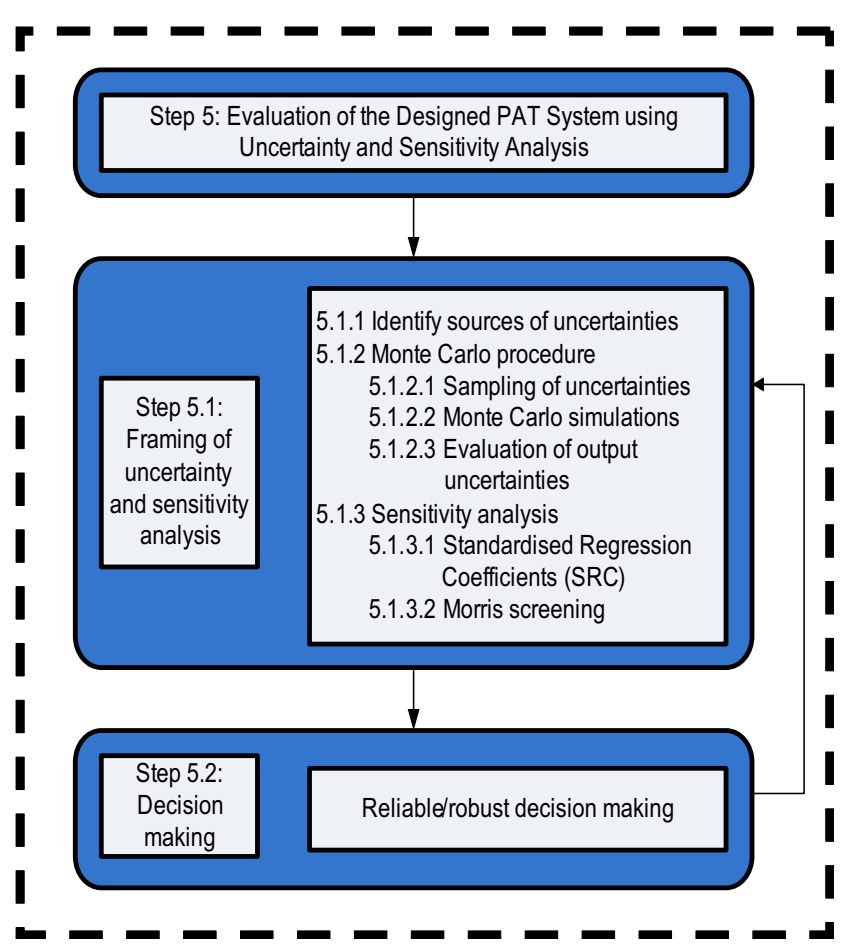

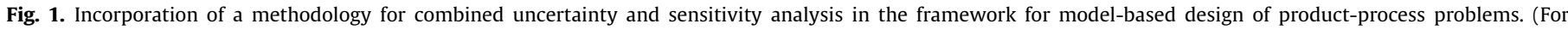
interpretation of the references to color in this figure legend, the reader is referred to the web version of this article.)

is indicated by the variance of the distribution, which indicates the spread of the data. In addition, a full characterization of uncertainty using an empirical cumulative distribution function (ECDF) is used and the probability of failure to reach a target CSD with a given tolerance/acceptable variation is calculated in order to determine the impact of input uncertainties on the model prediction. Here, the user needs to specify the minimum and maximum acceptable value of the mean CSD in order to determine the acceptable range of variation. In this work, the minimum acceptable value is indicated as the mean $\left(\mu_{\text {target }}\right)$ of the CSD minus one target standard deviation $\left(\sigma_{\text {target }}\right)$, and the maximum acceptable value is indicated as the mean $\left(\mu_{\text {target }}\right)$ of the CSD plus one target standard deviation $\left(\sigma_{\text {target }}\right)$.

\subsubsection{Sensitivity analysis (Step 5.1.3)}

The sensitivity analysis is performed next to identify the individual contributions of uncertain parameters to the total variance calculated in Step 5.1.2, which provides a parameter significance ranking for each output. As mentioned above, two methods namely (1) the Standardized regression coefficients (SRC) method [15,16]; and, (2) the Morris screening method [17-19] are used.

\subsubsection{Standardized regression coefficients (SRC) (Step 5.1.3.1). In} this method, a linear regression is performed on the Monte Carlo results describing each model output of interest as a multivariate linear function of the model inputs considered in the uncertainty analysis [15]. Here, the user selects the model output of interest to the analysis. For a crystallization process, the most important model output is usually the CSD, considering that this is one of the most important product quality specifications that need to be achieved. Therefore, the mean of the predicted CSD at the end of the crystallization process can be used as a scalar output for the SRC method. The scalar model output matrix can be denoted as sy and has the dimensions of $K \times N$ where $K$ is the number of output variables and $N$ is the number of samples. The regression model is then fitted to the (scalar) output of the Monte Carlo simulations relating model output, sy to the model inputs considered in the uncertainty analysis, $\theta_{i j}$ as shown in following equation:

$s y_{i k}=b_{0 k}+\sum_{j=1}^{M} b_{j k} \theta_{i j}+\varepsilon_{i k}$ for $i=1,2, \ldots, N$ and for $k=1,2, \ldots, K$

where $s y_{i k}$ is the scalar value for the $k$ th output, $b_{j k}$ is the coefficient of the $j$ th input parameter, $\theta_{j}$, for the $k$ th output, $\theta_{i j}$ is the value of the $j$ th parameter, and $\varepsilon_{i k}$ is the error of the regression model. Eq. (1) is then written in dimensionless form by scaling the outputs and the parameters using their corresponding means and standard deviations [15] as shown in following equation:

$\frac{s y_{i k}-\mu_{s y_{k}}}{\sigma_{s y_{k}}}=\sum_{j=1}^{M} \beta_{j k} \cdot \frac{\theta_{i j}-\mu_{\theta j}}{\sigma_{\theta j}}+\varepsilon_{i k}$

The standardized regression coefficients, $\beta_{j k}$, can have values in the range [-11] with the following meaning: (i) a high absolute value indicates a large effect of the corresponding parameter on the output, (ii) a negative sign indicates a negative effect and vice versa a positive sign indicates a positive effect on the output, and (iii) coefficients close to zero mean that the output is not sensitive to that parameter $[15,16]$. Furthermore, for these coefficients to be considered a valid measure of sensitivity, the coefficient of determination should be sufficiently high, $R^{2} \geqslant 0.7$, which implies that the relation between the parameters and the outputs is sufficiently linear $[15,18]$. In a sensitivity analysis context, the linear model determination coefficient, $R^{2}$, indicates what fraction of variance of the raw data obtained from the Monte Carlo simulations is explained by the linear model. For a meaningful sensitivity analysis, an $R^{2}$ value equal to or greater than 0.7 is considered as the minimum requirement, meaning that at least $70 \%$ of the output variance is explained by the linear model $[15,18]$. When $R^{2}$ is below this value, it means, from a sensitivity analysis point of view, that the linear model explains a too small fraction of the output variance and hence the SRC 
coefficients describe the parameter significance on outputs incompletely [23]. Or, in other words, the result of the SRC method is considered unreliable for $R^{2} \leqslant 0.7$ [15]. In this case, another global sensitivity analysis method should be used. To this end, we then recommend and employ the Morris screening method $[17,19]$ as an effective and reliable method for determining the most significant parameters.

2.1.3.2. Morris screening (Step 5.1.3.2). The Morris screening method relies on estimating the distribution of the elementary effects (EE) of each input parameter on the $k$ th model output called $E E_{j k}$ $[18,19]$. The $E E_{j k}$ attributable to each input parameter was obtained from the following differentiation of the model output, $s y_{k}$, with respect to the input, $\theta_{j}$, as shown in following equation:

$E E_{j k}=\frac{\partial s y_{k}}{\partial \theta_{j}}=\frac{s y_{k}\left(\theta_{1}, \theta_{2}, \theta_{j}+\Delta, \ldots, \theta_{M}\right)-s y_{k}\left(\theta_{1}, \theta_{2}, \theta_{j}, \ldots, \theta_{M}\right)}{\Delta}$

where $s y_{k}\left(\theta_{1}, \theta_{2}, \theta_{j}, \ldots, \theta_{M}\right)$ is the scalar model output evaluated at input parameters $\left(\theta_{1}, \theta_{2}, \theta_{j}, \ldots, \theta_{M}\right), s y_{k}\left(\theta_{1}, \theta_{2}, \theta_{j}+\Delta, \ldots, \theta_{M}\right)$ is the scalar model output corresponding to a $\Delta$ change in $\theta_{j}$, and $\Delta$ is a predetermined perturbation factor of $\theta_{j}$ and is calculated using the following equation $(\Delta=p /(2(p-1))$. The value of $p$ represents the level where the values should be taken for each input parameter, $\theta_{j}$. Here, the $p$ value can be equal to 4,6 , and 8 which corresponds to the $25 \mathrm{th}, 17 \mathrm{th}$, and 12.5 th percentile of input distributions. The distribution function is denoted as $F_{j k}$, which represents the distribution of the effects of the $j$ th input parameter on the $k$ th output. The $F_{j k}$ was estimated by performing calculations of the elementary effects, $E E_{j k}$, at randomly sampled points in the input space, and this procedure was repeated a number of times, $r$. In the Morris sampling design, the calculation of one elementary effect for each input parameter $M$ requires $(M+1)$ model simulations [19]. Because a number of repetitions, $r$, is needed (typically 10-50), the total number of model simulations needed for the Morris screening becomes $r^{*}(M+1)$. For the Morris method, there are three degrees of freedom that need to be specified which are the values of $\Delta, p$ and $r$, respectively. In this study, the values for $\Delta, p$, and $r$ were specified as $2 / 3,4$, and 10 , respectively. Finally, the Morris results can be evaluated by comparing the mean, $\mu_{j}$ and the standard deviations, $\sigma_{j}$ of the distribution functions, $F_{j k}$, of each input. The measure of sensitivity for the mean of the distribution functions follows the same concept as the standardized regression coefficients in the SRC method.

\subsection{Decision making (Step 5.2)}

In this step, the robustness of the model-based solution is evaluated by judging on a number of criteria including the probability of failure to meet the target CSD. As an example, a probability less than 0.05 could be assumed to indicate that a reliable PAT system to achieve the desired target CSD is obtained. In case the probability is exceeding the value of 0.05 , this then forms an indication that the PAT system needs to be improved further, for example by using a new controller structure or updated controller tuning parameters until an acceptable risk level (the probability of failure must be less than 0.05 ) is achieved.

\section{Application of the systematic framework for managing uncertainties: potassium dihydrogen phosphate (KDP) crystallization case study}

In our earlier study [1], it has been shown how the target oneand two-dimensional CSD for the potassium dihydrogen phosphate (KDP) crystallization process can be achieved systematically through the design framework. In our previous work, both a oneand a two-dimensional model were generated to represent the crystallization process. By implementing a controller (obtained through the PAT system design procedure) to maintain the KDP concentration at the required set-point, it was demonstrated that the target CSD was achieved for both the one- and the two-dimensional case. Here, the feature of the systematic design framework to perform uncertainty and sensitivity analysis on the designed PAT system is highlighted through the KDP crystallization process case study: it will be investigated how input (parameter) uncertainty affects the target one- and two-dimensional CSD and how this uncertainty can be minimized to achieve the desired target CSD. The target one- and two-dimensional CSD used are shown in Fig. 2 and can be described as follows: the mean characteristic length and standard deviation values for the target one-dimensional CSD are $60.85 \mu \mathrm{m}$ and $2.8 \mu \mathrm{m}$, respectively, while the mean characteristic length and width for the two-dimensional CSD are
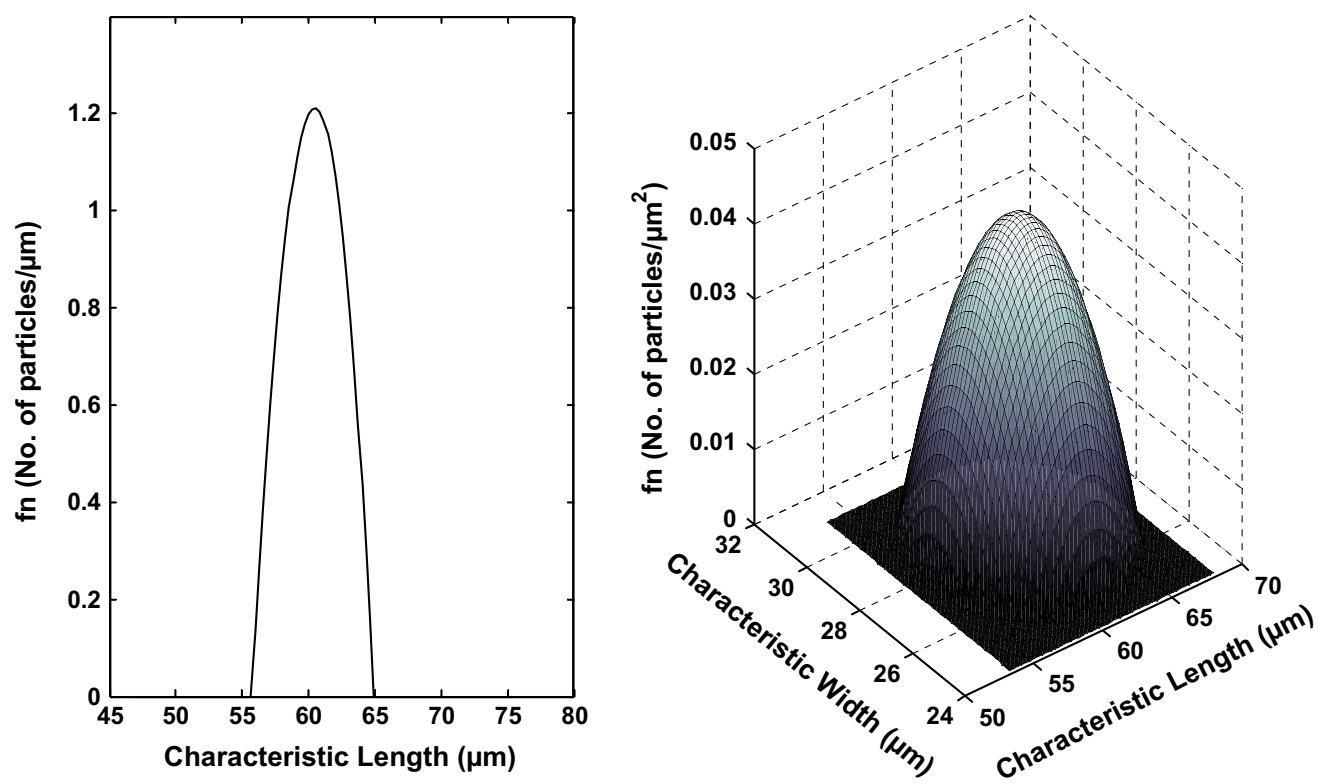

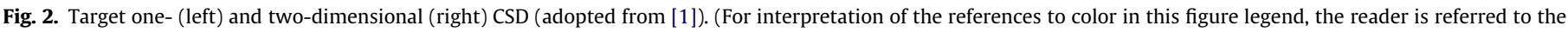
web version of this article.) 
set at $60.85 \mu \mathrm{m}$ and $27.36 \mu \mathrm{m}$ with a standard deviation of $2.8 \mu \mathrm{m}$ (length) and $1.34 \mu \mathrm{m}$ (width), respectively.

\subsection{Problem definition (Step 1)}

The main objective of this study is to develop a reliable and robust PAT system design for the KDP crystallization process by performing uncertainty and sensitivity analysis on the designed PAT system. In this study, the uncertainty and sensitivity analysis are performed under two different scenarios: (1) open-loop and (2) closed-loop.

\subsection{Crystallization model development (Step 2)}

Using the generic model-based framework [24], the relevant problem-chemical system specific models are obtained. In the one-dimensional model, size dependent growth, relative supersaturation, and a cube-shaped crystal are assumed to generate the problem-chemical system specific model. Table 1 shows the complete problem-chemical system specific one-dimensional model for the KDP crystallization process generated by the generic multi-dimensional model-based framework.

For the same chemical system and crystallization process, the one-dimensional model can be extended to a two-dimensional model $[1,24]$. The changes needed to develop the two-dimensional model of the KDP crystallization process are mainly the PBE formulation where the equations are now extended to consider growth in two directions (length and width). In the twodimensional model, a tetragonal prism-shaped crystal is assumed. The overall mass and energy balances are obtained by substituting the volume occupied by the selected crystal shape. In the constitutive equations, two crystal growth rate equations are now added, as well as equations for calculating the CSD, the total number of crystal particles, the total crystal mass, and the mean characteristic length and width. Other than that, the same assumptions, equations, and chemical properties used in the one-dimensional model are also used here. The generated problem-chemical system specific two-dimensional model equations are listed in the Appendix A.

\subsection{Generation of supersaturation set-point (Step 3)}

The supersaturation set-points that guarantee the target oneand two-dimensional CSDs are generated using an extended analytical CSD estimator [1]: the optimal supersaturation set-point that is to be maintained for both cases equals $0.03 \mathrm{~g} / \mathrm{g}$, with a total crystallization time of $80 \mathrm{~s}$ [1]. By using the optimal supersaturation set-point, the target one- and two-dimensional CSDs are indeed achieved, as reported in detail in our earlier work [1]. Here, the supersaturation set-point for the one- and two-dimensional KDP crystallization process is used for simulation under parametric uncertainties, and it is investigated for the closed-loop case how the set-point can be maintained under uncertainties in order to produce the desired target CSDs.

\subsection{Design of the PAT system (Step 4)}

The description of the PAT system designed for the KDP crystallization process is provided in Samad et al. [1] and is briefly summarized here. The basic raw materials required include: Water as a solvent and KDP as a solute assuming that the pure KDP has been isolated in water during the organic synthesis step. The process equipment used is a jacketed batch crystallizer. Based on a sensitivity analysis, it was concluded that the critical process variable is the KDP concentration and it can be controlled by manipulating either coolant flow rate or inlet water temperature. An interdependency analysis showed that the most suitable manipulated variable is the inlet water temperature due to the fact that a large change in the KDP concentration is obtained when the inlet water temperature is perturbed, compared to the relatively small change in the KDP concentration that results when the coolant flow rate is perturbed. Or, put differently, it is concluded that the inlet water temperature has most control authority with respect to the KDP concentration. The resulting proposed PAT system for the oneand two-dimensional KDP crystallization is indicated in Table 2, where a PI controller is implemented to control the KDP concentration by manipulating the inlet water temperature as shown in Fig. 3.

\subsection{Evaluation of the designed PAT system using uncertainty and sensitivity analysis (Step 5)}

In this study, the uncertainty and sensitivity analysis are performed under two different scenarios: (a) open-loop; and (b) closed-loop. The model implementations, the Monte Carlo simulations, and the Morris screening are all performed in the ICAS-MoT modeling tool [25].

Table 1

List of model equations for the one-dimensional model of KDP crystallization.

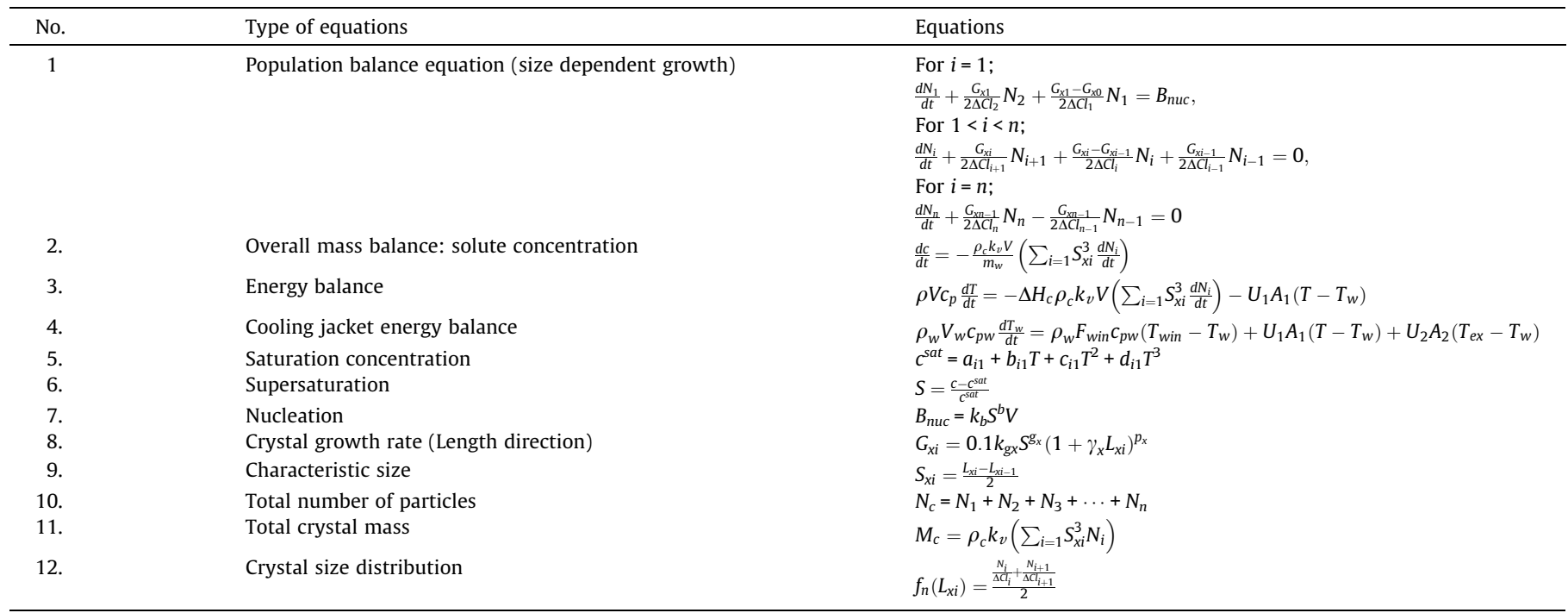




\subsubsection{Open-loop}

The objective here is to understand the effect of uncertain system parameters such as nucleation and crystal growth parameters on the prediction of the crystallization performance and to identify which parameters are responsible for the variance in the product CSD. Furthermore, the open-loop results will form the reference for comparison with the results obtained from closed-loop simulations using the designed PAT system. The open-loop analysis is conducted separately for the one-dimensional and the two-dimensional KDP crystallization process model, where the model equations used are shown in Table 1 and Appendix A, respectively. The resulting performance is evaluated here in terms of KDP concentration, temperature profile, and final CSD for both cases. The evaluation is first done assuming no uncertainty on the inputs, i.e., only a single output profile is obtained for each output variable. The open-loop reference simulation results for the one- and twodimensional KDP crystallization process are shown in Fig. 4.

A seeded crystallization process is studied, where the CSD of the initial crystal seeds added to the solution is expected to grow into the desired target CSD using natural cooling profiles. The temperature is reduced from $32{ }^{\circ} \mathrm{C}$ to $28^{\circ} \mathrm{C}$, resulting in KDP concentration profiles that are in both cases (one-dimensional as well as twodimensional) deviating considerably from the saturation concentration line. Under such conditions, a too high supersaturation is obtained in the beginning of the operation and therefore undesired secondary nucleation occurs. The final one- and two-dimensional
CSDs (shown as contour plot) are shown in Fig. 4 (bottom row). The CSDs indicate that the initially added crystal seeds have grown, as expected. However, in addition to this, there is also a secondary peak visible, consisting of small crystals that were formed due to nucleation, as a direct result of a too high supersaturation.

\section{Step 5.1: Framing for uncertainty and sensitivity analysis}

The framing scenario for uncertainty and sensitivity analysis is as follows for the KDP crystallization process:

\section{Step 5.1.1: Identify sources of uncertainty}

In this work, five parameters $\left(k_{b}, b, k_{g x}, g_{x}\right.$, and $\left.\gamma_{x}\right)$ from the nucleation and crystal growth model equations (see Eqs. (7) and (8) in Table 1) were investigated for the one-dimensional case and 8 parameters $\left(k_{b}, b, k_{g x}, g_{x}, \gamma_{x}, k_{g y}, g_{y}\right.$ and $\left.\gamma_{y}\right)$ from the nucleation and crystal growth models (see Eqs. (7)-(9)) in Appendix A) were investigated for the two-dimensional case. All the parameters are assumed to have a uniform probability distribution as shown in Table 3 where the reported lower and upper bound values of each parameter are obtained based on the $95 \%$ confidence intervals taken from Gunawan et al. [26,27]. However, the confidence interval for both growth constants in the length and width direction is assumed in this study. The kinetic parameters for nucleation and crystal growth rate are usually specific for the system that is

Table 2

Proposed process monitoring and control (PAT) system for one- and two-dimensional KDP crystallization (adopted from [1]).

\begin{tabular}{|c|c|c|c|c|}
\hline \multicolumn{5}{|c|}{ Proposed design of PAT system } \\
\hline $\begin{array}{l}\text { Critical } \\
\text { process } \\
\text { points }\end{array}$ & $\begin{array}{l}\text { Critical process } \\
\text { variables }\end{array}$ & Actuators & Monitoring techniques & Monitoring tools \\
\hline Crystallizer & Concentration & $\begin{array}{l}\text { Inlet water } \\
\text { temperature }\end{array}$ & ATR-FTIR & ATR-FTIR probe \\
\hline Crystallizer & Temperature & $\begin{array}{l}\text { Inlet water } \\
\text { temperature }\end{array}$ & Thermocouple & WZ-08541-28 (E20-gauge thermocouple) \\
\hline Crystallizer & $\begin{array}{l}\text { Crystal size } \\
\text { distribution (CSD) }\end{array}$ & - & $\begin{array}{l}\text { Laser diffraction (one-dimensional); In situ } \\
\text { video microscopy (two-dimensional) }\end{array}$ & $\begin{array}{l}\text { Malvern Mastersizer (one-dimensional); Mettler-Toledo } \\
\text { particle vision microscope (two-dimensional) }\end{array}$ \\
\hline
\end{tabular}

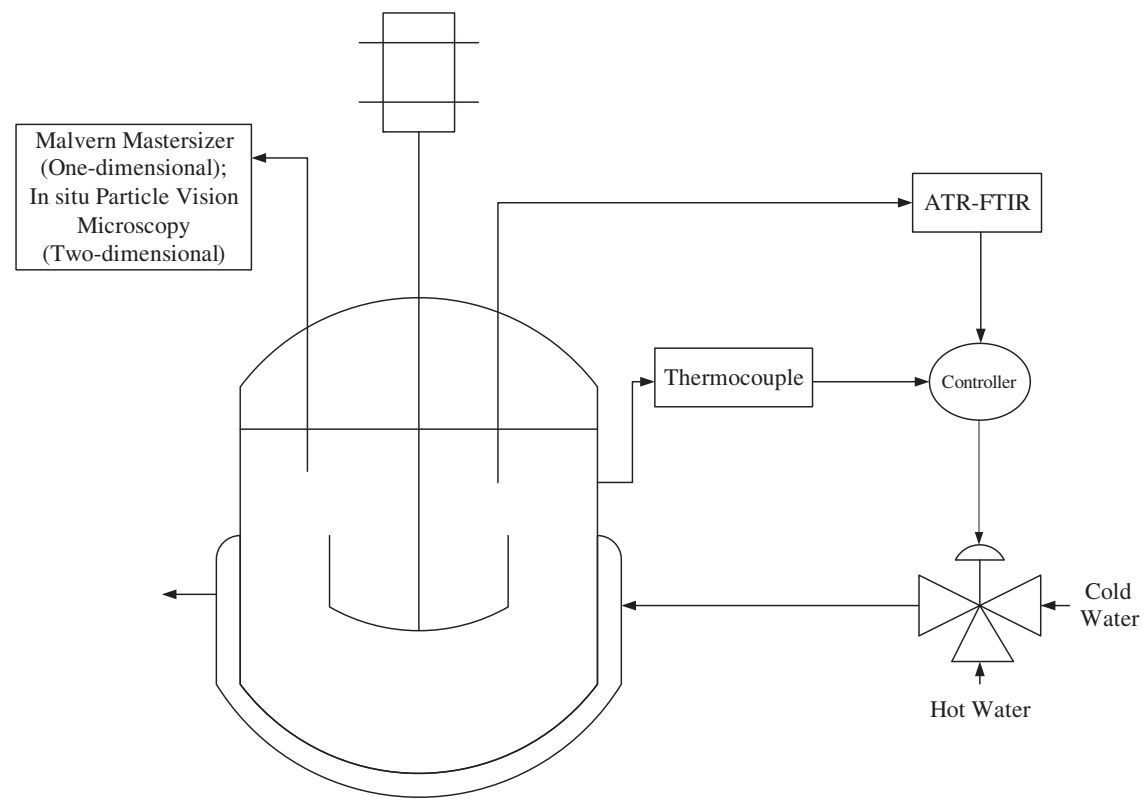

Fig. 3. KDP crystallization process flowsheet with designed PAT system. 

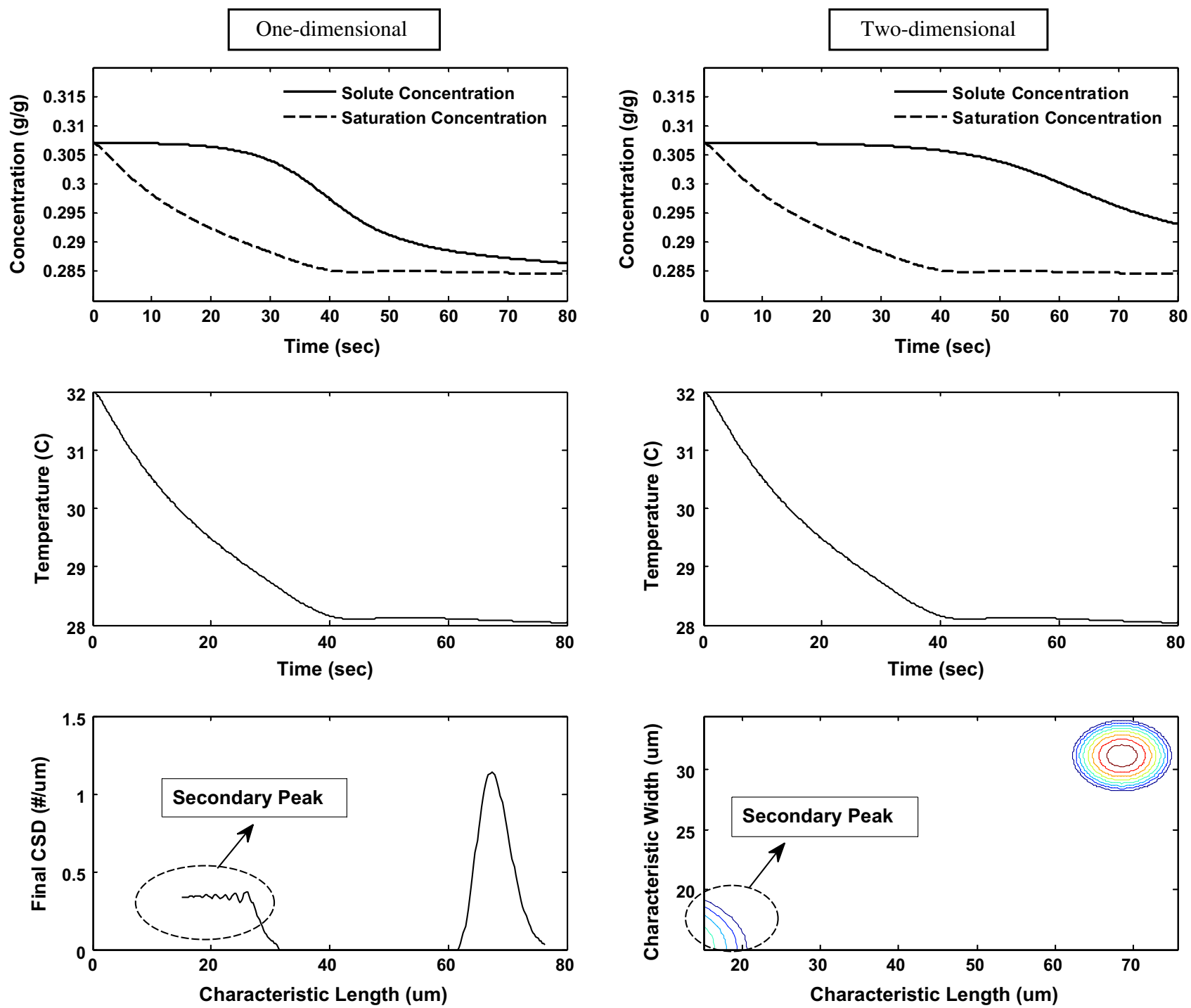

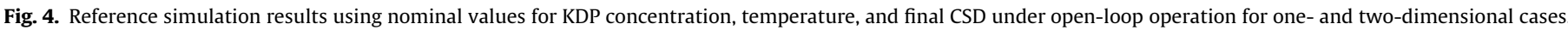
(For interpretation of the references to color in this figure legend, the reader is referred to the web version of this article.)

studied, and in this work, the parameters were chosen based on the fact that they have been estimated from experimental KDP crystallization data [26]. The use of a different set of parameters, for example when studying another chemical system, will of course influence the nucleation and crystal growth rate and thus also the resulting CSD.

\section{Step 5.1.2: Monte Carlo procedure}

The first step in the Monte Carlo procedure is the sampling of uncertainties (Step 5.1.2.1). In this step, a parameter is sampled from a distribution using the Latin-hypercube sampling (LHS) method. Different numbers of samples have been tested $(25,50$, 100 , and 150 samples) and were compared based on the Monte Carlo simulation errors to determine whether the number of samples used is suitable or not. The analysis showed that the errors for 25 and 50 samples are larger than the error for 100 samples. However, the error for 100 and 150 samples is almost identical. Therefore, the number of samples used in this study is 100 samples for both the one- and the two-dimensional cases when no correlation between the parameters is assumed. The next step in the Monte Carlo procedure is to perform simulations (Step 5.1.2.2). In this

Table 3

Input uncertainties on nucleation and crystal growth rate parameters for one- and two-dimensional KDP crystallization (adopted from [26,27]).

\begin{tabular}{|c|c|c|c|c|c|}
\hline Parameters & Units & Values & Confidence interval (95\%) & Lower bound values & Upper bound values \\
\hline Nucleation rate constant, $k_{b}$ & No. of particles $/ \mu \mathrm{m}^{3} \mathrm{~s}$ & $7.49 \mathrm{E}-08$ & $\pm 3.5 \mathrm{E}-09$ & $7.14 \mathrm{E}-08$ & $7.84 \mathrm{E}-08$ \\
\hline Nucleation order constant, $b$ & Dimension-less & 2.04 & \pm 0.16 & 1.88 & 2.2 \\
\hline Growth rate constant (length direction), $k_{g x}$ & $\mu \mathrm{m} / \mathrm{s}$ & 100.75 & \pm 12.3833 & 88.3667 & 113.1333 \\
\hline Growth order (length direction) constant, $g_{x}$ & Dimension-less & 1.74 & \pm 0.07 & 1.67 & 1.81 \\
\hline Growth constant (length direction), $\gamma_{x}$ & $1 / \mu \mathrm{m}$ & 0.6 & \pm 0.1 & 0.5 & 0.7 \\
\hline Growth rate constant (width direction), $k_{g y}$ & $\mu \mathrm{m} / \mathrm{s}$ & 12.21 & \pm 3.3167 & 8.8933 & 15.5267 \\
\hline Growth order constant (width direction), $g_{y}$ & Dimension-less & 1.48 & \pm 0.06 & 1.42 & 1.54 \\
\hline Growth constant (width direction), $\gamma_{y}$ & $1 / \mu \mathrm{m}$ & 0.6 & \pm 0.1 & 0.5 & 0.7 \\
\hline
\end{tabular}


case, the open-loop one- and two-dimensional KDP models are simulated 100 times, i.e., once for each different set of model parameters that was sampled.

The results from the Monte Carlo simulations are then analyzed in the evaluation of output uncertainties step (Step 5.1.2.3) as shown in Fig. 5. Each line in Fig. 5, for example for the final CSD, corresponds to a dynamic model output obtained by simulating the one- and two-dimensional KDP model with one set of parameter values (one sample). For both the one- and the two-dimensional case, the parametric uncertainties (nucleation and crystal growth rate parameters) are clearly affecting the simulated KDP concentration profiles and the final one- and two-dimensional CSD (shown as contour plot). Meanwhile, the corresponding oneand two-dimensional temperature profiles are less affected by the input uncertainties.

In this study, we have found that the output uncertainty in the two-dimensional case is more pronounced compared to the onedimensional case. While not generalizing to other chemical systems, the underlying reason may be due to the fact that different crystal shapes are used in both cases (cube-shaped for the onedimensional case and tetragonal prism-shaped for the two-dimensional case). For the cube-shaped crystals, only a single crystal growth model is needed because we assume that the crystal is grown at the same rate in all directions. However, two crystal growth rate models (length and width) are needed to cover the growth of a tetragonal prism-shaped crystal. The crystal growth model is also related to the KDP concentration equation as well as the CSD through the population balance equation which explains the spread of these model outputs. Usually, the kinetic parameters for the crystal growth model are obtained from experimental measurements, and this implies a certain error on the estimated parameters. Consequently, the use of two crystal growth models in the two-dimensional case most probably contributes more to the uncertainty in the KDP concentration and CSD model prediction compared to the one-dimensional case.

The data can be assessed further using the mean, 10th, and 90th percentiles of the Monte Carlo output at each simulation time as indicated in Fig. 6. Based on Fig. 6, the 10th and 90th percentile for the temperature in both the one- and the two-dimensional case are very close to the mean, indicating that the uncertainty of this model output is very low or not existing in open-loop. However, the 10th and 90th percentiles for the KDP concentration and final CSD for both cases are further away from the mean. Based on this analysis, the risk of not achieving the target CSD is calculated for both open-loop cases (one-dimensional and two-dimensional, respectively). As mentioned earlier, the mean characteristic length
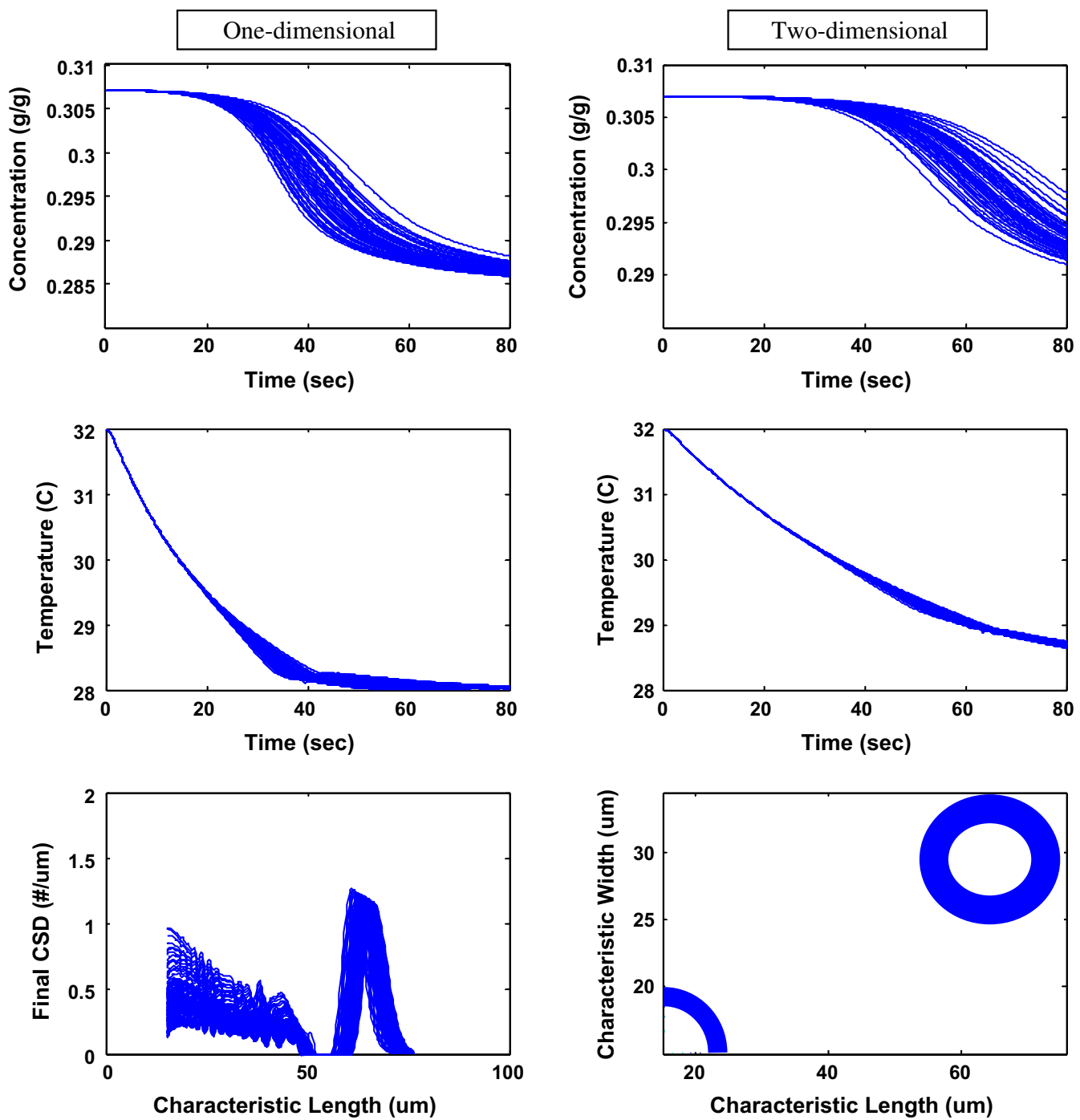

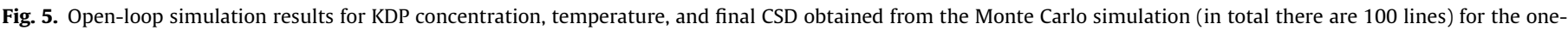
and two-dimensional cases. (For interpretation of the references to color in this figure legend, the reader is referred to the web version of this article.) 
of the target CSD for the one-dimensional case is $60.85 \mu \mathrm{m}$. Meanwhile, for the two-dimensional case, the mean characteristic length and width are specified as $60.85 \mu \mathrm{m}$ and $27.36 \mu \mathrm{m}$, respectively. By using this information, the mean values of the target CSDs are 1.22 no. of particles/ $\mu \mathrm{m}$ and 0.043 no. of particles $/ \mu \mathrm{m}^{2}$, respectively (see Fig. 2). For the purpose of highlighting the calculation of the risk associated with a given PAT design, defined as the probability of failure to reach a target CSD envelope, the target envelope for the CSD is then assumed as $1.22 \pm 0.04$ no. of particles $/ \mu \mathrm{m}$ for the one-dimensional case (minimum acceptable CSD is 1.18 no. of particles/ $\mu \mathrm{m}$ and maximum acceptable CSD is 1.26 no. of particles $/ \mu \mathrm{m})$. For the two-dimensional case, the target envelope for the CSD is assumed as $0.043 \pm 0.005$ no. of particles/ $\mu \mathrm{m}^{2}$ (minimum acceptable CSD is 0.038 no. of particles/ $\mu \mathrm{m}^{2}$ and maximum acceptable CSD is 0.048 no. of particles $/ \mu \mathrm{m}^{2}$ ). As shown in Fig. 7, the probability for the one-dimensional case of not achieving the acceptable CSD (i.e., the probability of achieving a CSD below the mean - standard deviation threshold in this case) is 0.75 . Meanwhile, a probability of not achieving the CSD equal to 0.8 is obtained for the two-dimensional case. This is not surprising, as in the open-loop conditions, there is no PAT system/control system established to ensure that the target CSD is achieved.

\section{Step 5.1.3: Sensitivity analysis}

In this step, the sensitivity analysis is conducted first using the SRC method. Then, the reliability of the parameter significance ranking obtained by the SRC method is confirmed through the use of the Morris screening method.

Step 5.1.3.1: Standardized regression coefficients (SRC) method

The sensitivity analysis is conducted for two different points in the simulated one-dimensional CSD data, as shown in Fig. 8. One data point in the CSD corresponds to the crystals formed due to secondary nucleation (point p1 in Fig. 8) and the other one corresponds to the CSD from the seeded crystals that have grown (point p2 in Fig. 8). For each data point, a linear regression model was constructed using Eq. (1) and the corresponding regression
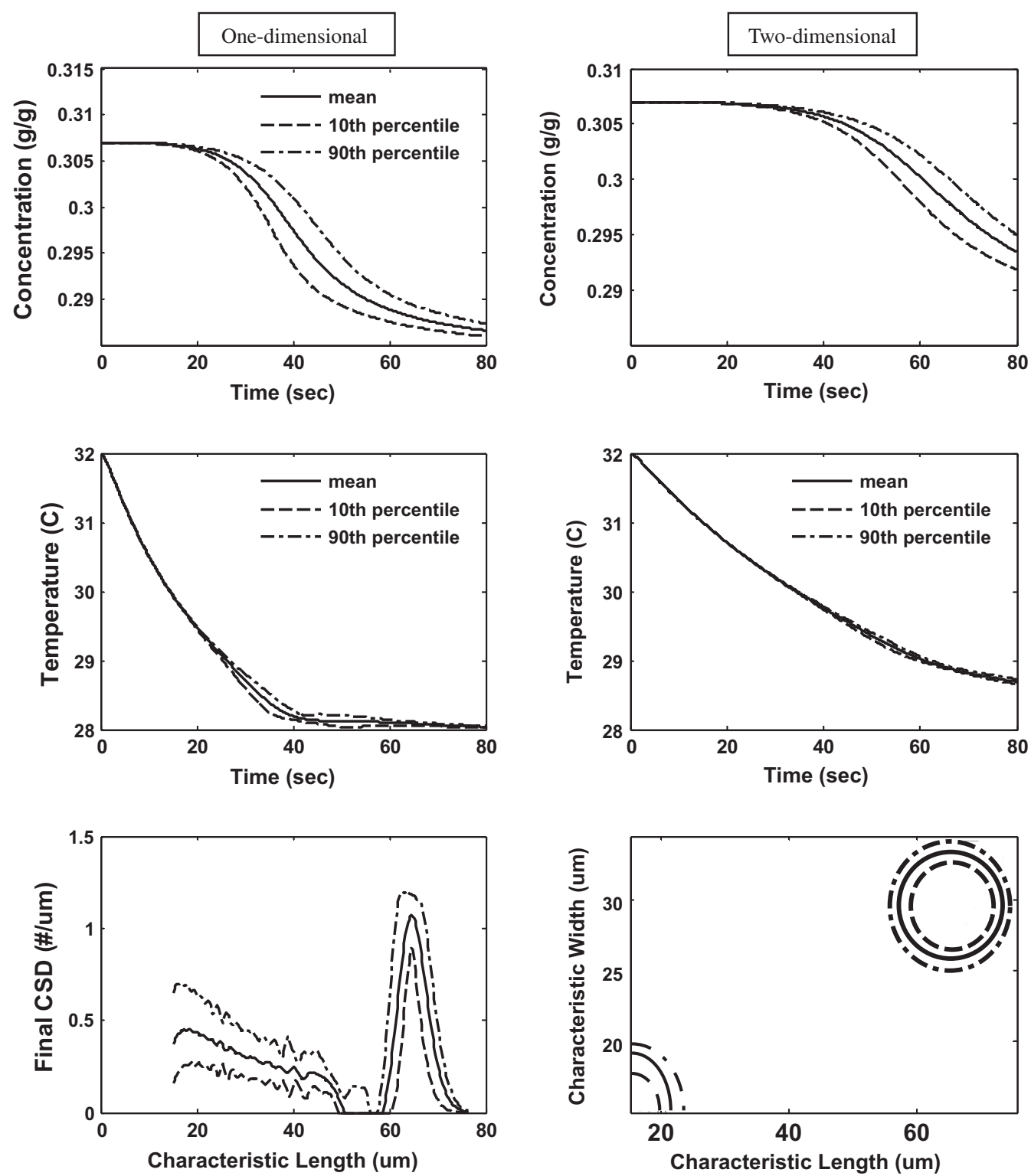

Fig. 6. Representation of uncertainty using mean, 10th, and 90th percentile values of the Monte Carlo simulations under open-loop conditions. 

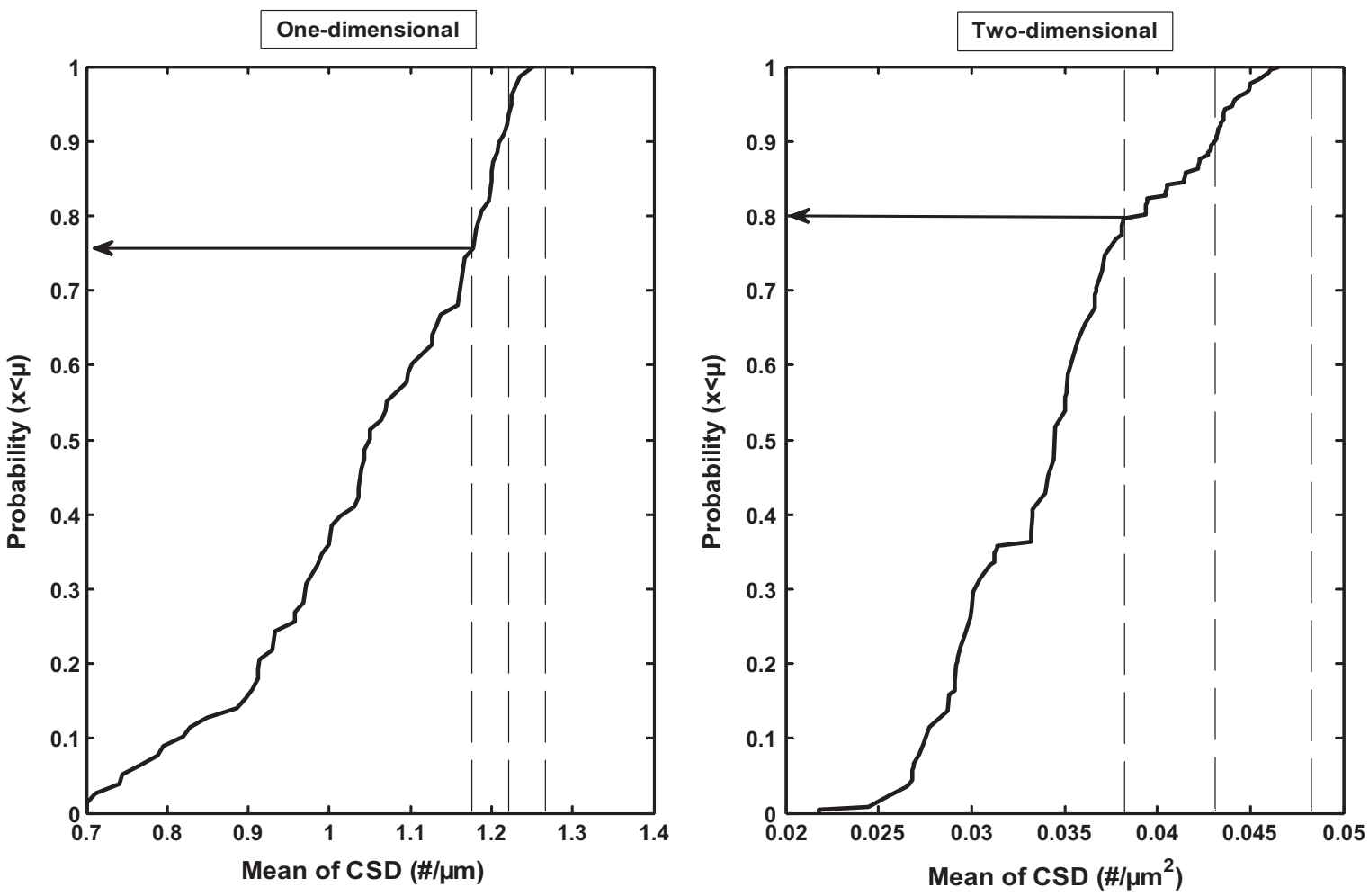

Fig. 7. Risk of not achieving the desired target for the one- and two-dimensional cases in open-loop operation.

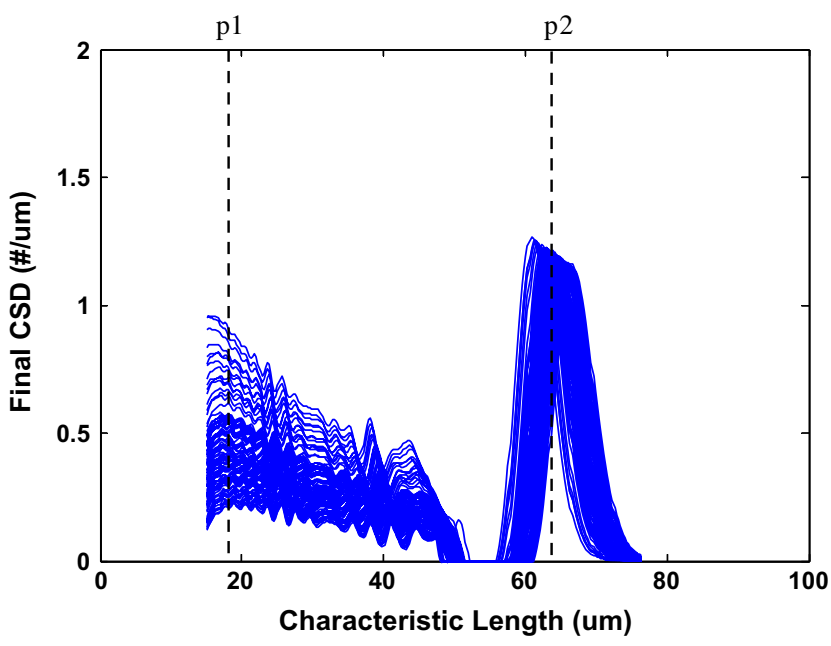

Fig. 8. Points where the one-dimensional CSD is sampled for sensitivity analysis. (For interpretation of the references to color in this figure legend, the reader is referred to the web version of this article.)

Table 4

Standardized regression coefficients of linear models and parameter significance ranking for the one-dimensional case.

\begin{tabular}{lllllr}
\hline \multirow{2}{*}{ Location } & \multicolumn{2}{l}{ CSD data taken at point $\mathrm{p} 1$} & & \multicolumn{2}{l}{ CSD data taken at point $\mathrm{p} 2$} \\
& $R^{2}=0.9506$ & & & $R^{2}=0.8456$ & \\
\cline { 2 - 3 } \cline { 5 - 6 } Ranking & Parameter & $\beta_{j k}$ & & Parameter & $\beta_{j k}$ \\
\hline 1 & $b$ & -0.8241 & & $g_{x}$ & 0.6890 \\
2 & $g_{x}$ & 0.4502 & & $\gamma_{x}$ & -0.5804 \\
3 & $\gamma_{x}$ & -0.2355 & & $k_{g x}$ & 0.3405 \\
4 & $k_{g x}$ & -0.1734 & & $b$ & -0.1603 \\
5 & $k_{b}$ & 0.063 & & $k_{b}$ & -0.03 \\
\hline
\end{tabular}

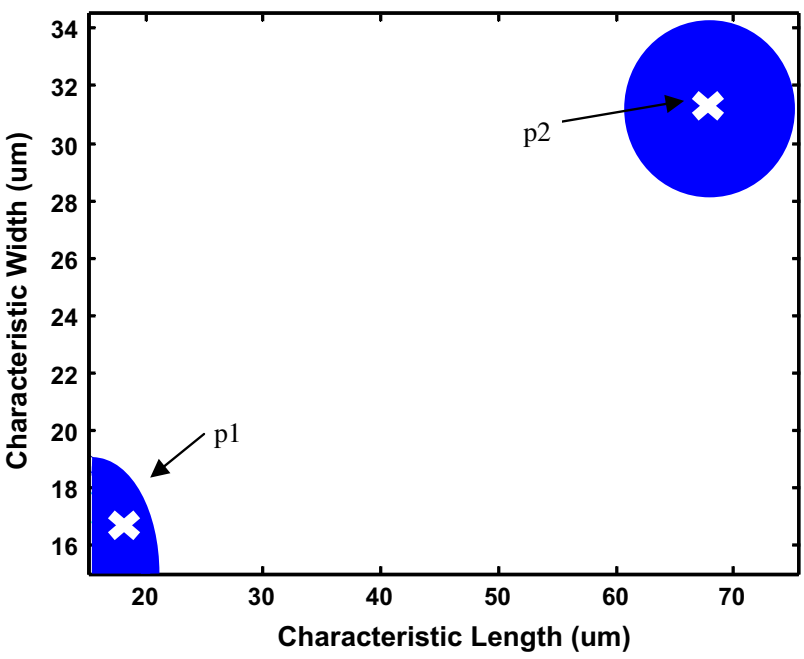

Fig. 9. Points where the two-dimensional CSD is sampled for sensitivity analysis. (For interpretation of the references to color in this figure legend, the reader is referred to the web version of this article.)

coefficients, $\alpha_{j k}$, were obtained from linear least-squares. The standardized regression coefficients, $\beta_{j k}$, were then calculated using Eq. (2), and the resulting summary of the parameter significance ranking for the one-dimensional case is given in Table 4 .

The degree of linearization indicated by the coefficient of model determination, $R^{2}$, was found to be high for all CSD data taken, i.e., the $R^{2}$ values were above the recommended value of 0.7 $[15,18]$. This indicates that the linearized model is reliable and the corresponding coefficients can be used to assess and rank the importance of the input parameters with respect to the outputs. Based on the one-dimensional CSD data taken at p1, it was shown that the most significant parameter is the nucleation 
Table 5

Standardized regression coefficients of linear models and parameter significance ranking for the two-dimensional case.

\begin{tabular}{lllllr}
\hline \multirow{2}{*}{ Location } & \multicolumn{2}{l}{ CSD data taken at point $\mathrm{p} 1$} & & \multicolumn{2}{l}{ CSD data taken at point $\mathrm{p} 2$} \\
& \multicolumn{2}{l}{$R^{2}=0.9212$} & & & $R^{2}=0.8443$ \\
\cline { 2 - 3 } \cline { 5 - 6 } Ranking & Parameter & $\beta_{j k}$ & & Parameter & $\beta_{j k}$ \\
\hline 1 & $b$ & -0.9032 & & $g_{x}$ & 0.6641 \\
2 & $g_{x}$ & 0.2312 & & $g_{y}$ & 0.5395 \\
3 & $g_{y}$ & 0.1932 & & $\gamma_{x}$ & -0.3781 \\
4 & $\gamma_{x}$ & -0.1671 & & $\gamma_{y}$ & -0.3021 \\
5 & $\gamma_{y}$ & -0.0921 & & $k_{g x}$ & 0.1643 \\
6 & $k_{b}$ & 0.0851 & & $k_{g y}$ & 0.1296 \\
7 & $k_{g x}$ & -0.0212 & & $b$ & -0.0403 \\
8 & $k_{g y}$ & -0.0128 & & $k_{b}$ & -0.0276 \\
\hline
\end{tabular}

order constant, $b$, which has a SRC of -0.82 and belongs to the nucleation equation (Eq. (7) in Table 1). This is a reasonable result, considering that the data are taken from the CSD region generated by secondary nucleation. Therefore, the variation of the input parameter, $b$, influences the generation of new crystals due to nucleation effects, which explains the large variation of the CSD data taken at $\mathrm{p} 1$. In addition, the negative sign for the parameter, $b$, indicates the negative impact on the CSD where more new crystals are generated when the value of the parameter, $b$, is decreased.

Meanwhile, the parameters, $g_{x}$ and $\gamma_{x}$, have an SRC of 0.45 and -0.24 , respectively. Both parameters are part of the crystal growth rate equation (Eq. (8) in Table 1) which also contributes to the large uncertainty on the CSD data prediction at point $\mathrm{p} 1$. Both parameters will have an effect on the growth of the new crystals that have been produced due to the secondary nucleation where the parameter, $g_{x}$, is responsible for driving the CSD toward a larger characteristic length, whereas the parameter, $\gamma_{x}$, contributes to variation of the secondary peak of the CSD data at point $\mathrm{p} 1$. In this case, the parameter, $\gamma_{x}$, has a negative impact, i.e., the lower the value of parameter, $\gamma_{x}$, the higher the secondary peak that will be obtained, which also helps to explain the large variation of the CSD data at point $\mathrm{p} 1$. The one-dimensional CSD data taken at $\mathrm{p} 2$ correspond to crystal growth of the original seed crystals. The most significant parameters for the data taken at p2 are $g_{x}, \gamma_{x}$, and $k_{g x}$ with SRCs of $0.69,-0.58$, and 0.34 , respectively. All three parameters appear in the crystal growth rate equation (Eq. (8) in Table 1). A higher value of the growth order constant $\left(g_{x}\right)$ results in a CSD with a higher characteristic length, which explains the variation in the distribution of the CSD data. Table 4 shows that the magnitude of the SRC for the growth constant $\left(\gamma_{x}\right)$ is negative. It means that when the value of the growth constant $\left(\gamma_{x}\right)$ is decreased, a higher CSD peak is obtained resulting into a narrower CSD as well. The nucleation phenomenon has no influence on the CSD data taken at $\mathrm{p} 2$ which explains why both parameters for nucleation now have the lowest ranking in the table.

Similarly, the sensitivity analysis was also conducted for two different parts of the two-dimensional CSD data, which are shown as a contour plot in Fig. 9: the data of p1 correspond to the part of the CSD generated by secondary nucleation, and the data of p2 are located in the center of the part of the CSD that has originated from the growth of the seeded crystals. The sensitivity analysis showed that the most significant parameters obtained for the two different locations in the two-dimensional case are identical to the most significant parameters that were found for the one-dimensional case. The main difference is that there are two different crystal growth rates used in the twodimensional case.

Based on Table 5, it is clear that the parameters corresponding to the crystal growth rate for the characteristic length direction are more significant than the parameters corresponding to the crystal growth rate for the characteristic width direction. For example, the most significant parameter for data taken at $\mathrm{p} 2$, the parameter $g_{x}$ for the characteristic length direction is more influential compared to the parameter $g_{y}$ for the characteristic width direction. This is because the parameters in the crystal growth expression for the characteristic length direction have a higher value than the parameters in the crystal growth model for the characteristic width direction and, thereby, contribute with more uncertainty to the model prediction. This is due to the fact that usually the high characteristic length is favorable in the twodimensional case in order to achieve a desired aspect ratio (average characteristic length over average characteristic width) around $1.5-2.2[28]$.

\section{Step 5.1.3.2: Morris screening method}

In this work, the parameter significance ranking obtained from the Morris screening method has been compared with the one obtained from the SRC method to investigate the reliability of both sensitivity analysis results. Firstly, the parameter significance ranking has been compared based on the one-dimensional CSD data taken at p1 and p2 (refer to Fig. 8). The results of the Morris method were found to be in good agreement for all the parameters with the ranking obtained by the SRC method as shown in Tables 6 and 7. Similarly, for the two-dimensional CSD data, the parameter significance ranking obtained from the Morris screening method is also identical to the ranking obtained by the SRC method as shown in Appendix B.

\subsubsection{Closed-loop}

The open-loop analysis for the one- and two-dimensional KDP crystallization process concluded that significant uncertainty exists in the model outputs for both cases, especially for the KDP concentration and the final CSD. The most significant parameter for that part of the CSD data generated by secondary nucleation is the nucleation order constant, $b$, for both cases. Meanwhile, the growth order constant, $g_{x}$, is identified as the most significant parameter for the part of the CSD corresponding to the seeded crystals for both cases respectively. With this result in mind, we now repeat the uncertainty and sensitivity analysis for the designed PAT system, i.e., we perform closed-loop simulation. The objective of the closed-loop analysis is to comprehensively test

Table 6

Method comparison for screening influential factors based on the one-dimensional CSD data taken at $\mathrm{p} 1$.

\begin{tabular}{llllll}
\hline \multirow{2}{*}{ Ranking } & \multicolumn{2}{l}{ Morris screening method } & & \multicolumn{2}{l}{ SRC method } \\
\cline { 2 - 3 } \cline { 5 - 6 } \cline { 5 - 6 } & Parameters & $\mu_{j}$ & & Parameters & \multicolumn{1}{l}{$\beta_{j k}$} \\
\hline 1 & $b$ & -0.8213 & & $b$ & -0.8241 \\
2 & $g_{x}$ & 0.3912 & & $g_{x}$ & 0.4502 \\
3 & $\gamma_{x}$ & -0.2687 & & $\gamma_{x}$ & -0.2355 \\
4 & $k_{g x}$ & -0.1154 & & $k_{g x}$ & -0.1734 \\
5 & $k_{b}$ & 0.0294 & & $k_{b}$ & 0.063 \\
\hline
\end{tabular}

Table 7

Method comparison for screening influential factors based on the one-dimensional CSD data taken at p2.

\begin{tabular}{lllllr}
\hline \multirow{2}{*}{ Ranking } & \multicolumn{2}{l}{ Morris screening method } & & \multicolumn{2}{l}{ SRC method } \\
\cline { 2 - 3 } & Parameters & $\mu_{j}$ & & Parameters & \multicolumn{1}{l}{$\beta_{j k}$} \\
\hline 1 & $g_{x}$ & 0.6623 & & $g_{x}$ & 0.6890 \\
2 & $\gamma_{x}$ & -0.5456 & & $\gamma_{x}$ & -0.5804 \\
3 & $k_{g x}$ & 0.4312 & & $k_{g x}$ & 0.3405 \\
4 & $b$ & -0.1221 & & $b$ & -0.1603 \\
5 & $k_{b}$ & -0.0335 & & $k_{b}$ & -0.03 \\
\hline
\end{tabular}


the PAT system design performance in achieving the desired target product properties taking into account the considered domain of uncertainties. For the closed-loop simulation, PI controllers have been developed for both the one- and the two-dimensional case; the controlled variable is the KDP concentration and the manipulated variable is the inlet water temperature for both cases. The controller objective is to maintain the KDP concentration at the generated supersaturation set-point of $0.03 \mathrm{~g} / \mathrm{g}$ during the total crystallization time $(80 \mathrm{~s})$ for both the one- and the two-dimensional case. The supersaturation of $0.03 \mathrm{~g} / \mathrm{g}$ is the distance between the actual KDP concentration and the solubility line that needs to be maintained until the end of the crystallization in order to achieve the target CSD [1]. On the basis of the concentration versus temperature phase diagram, such a supersaturation then translates into a corresponding temperature set-point profile that is to be followed by the controller. The closed-loop simulation results obtained for the one- and two-dimensional cases are shown in Fig. 10 for the nominal parameter values.
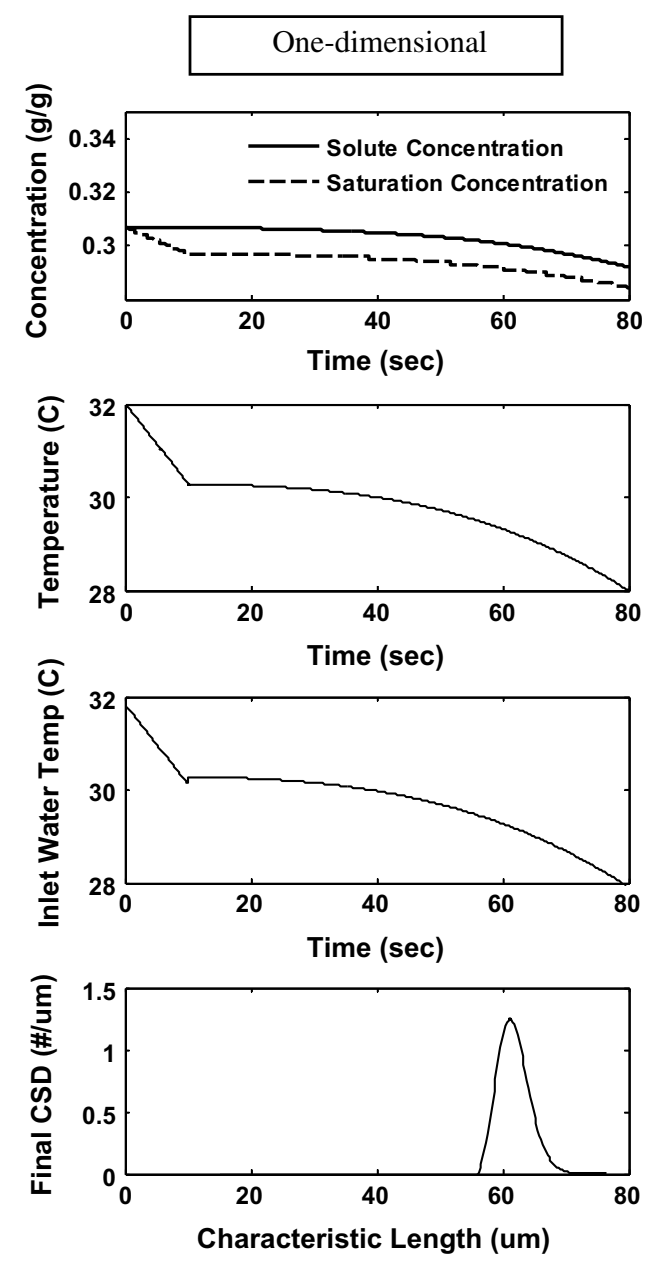

The KDP concentration for both cases initially started at $0.307 \mathrm{~g}$ $\mathrm{KDP} / \mathrm{g}$ water, and once the KDP concentration set-point was reached, the PI controller successfully maintained the concentration at the set-point until the end of the operation. In Fig. 10 (first row), approximately $0.2921 \mathrm{~g} \mathrm{KDP} / \mathrm{g}$ water (one-dimensional) and $0.2957 \mathrm{~g} \mathrm{KDP} / \mathrm{g}$ water (two-dimensional) remains by the end of the operation. The temperature profile (see second row in Fig. 10) corresponding to the generated supersaturation set-point starts for both cases at $32{ }^{\circ} \mathrm{C}$. The batch crystallizer is first cooled down relatively fast to about $30.3^{\circ} \mathrm{C}$ to reach the supersaturation set-point. From then onwards, the temperature in the one-dimensional case (final value $28^{\circ} \mathrm{C}$ ) is decreasing faster than in the two-dimensional case (final value $28.7^{\circ} \mathrm{C}$ ). In terms of CSD (see fourth row in Fig. 10), the detailed one-dimensional simulation model predicted a CSD that is almost identical to the target CSD. The cube-shaped seed crystals originally at mean characteristic length of $19.5 \mu \mathrm{m}$ with a standard deviation of $0.97 \mu \mathrm{m}$ have been grown to a mean characteristic length of $60.73 \mu \mathrm{m}$ with a standard deviation of $2.79 \mu \mathrm{m}$ which is very close to the mean characteristic length of
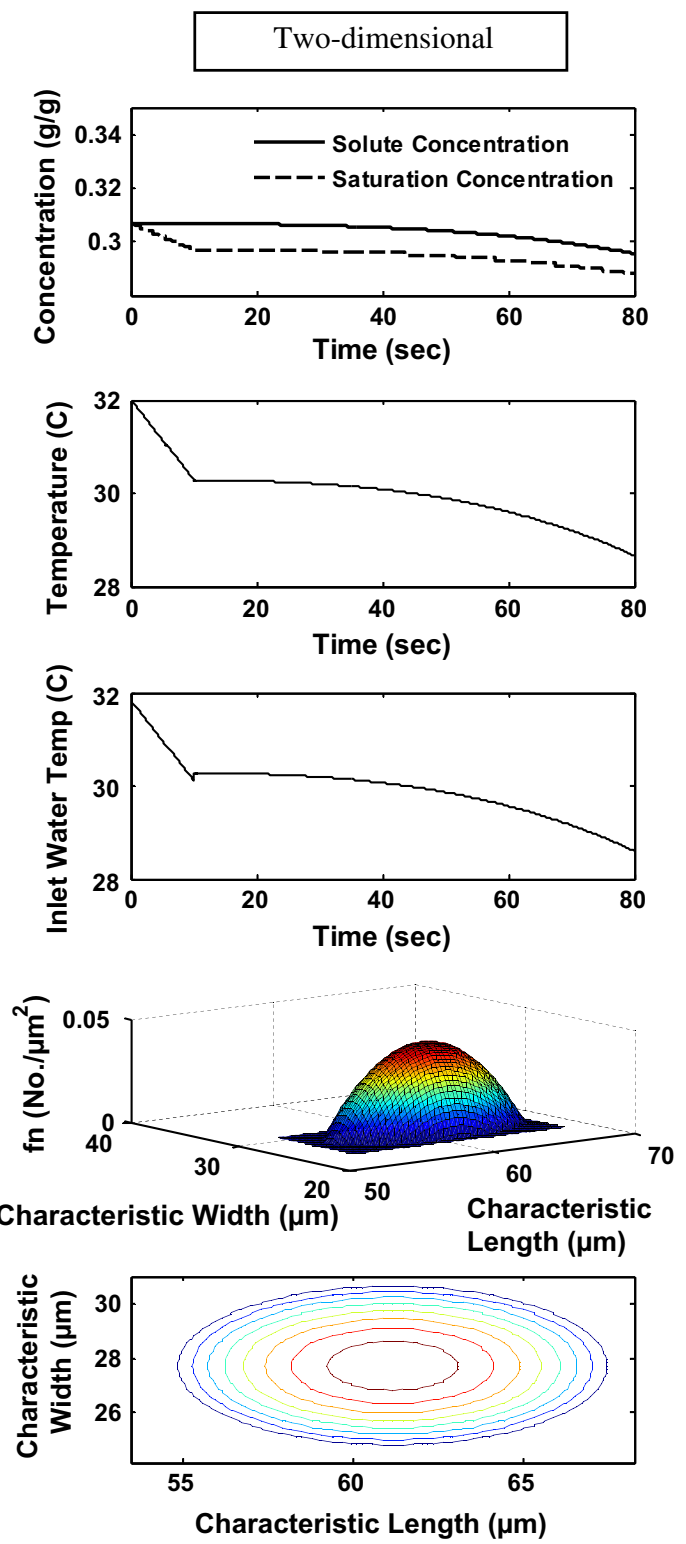

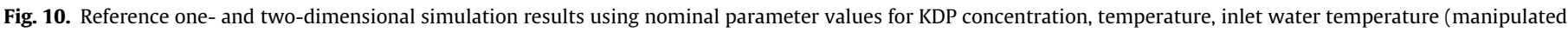

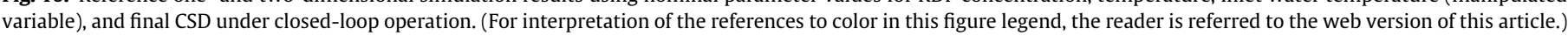


$60.85 \mu \mathrm{m}$ and standard deviation of $2.8 \mu \mathrm{m}$ for the target CSD. In the two-dimensional case, the final CSD obtained from the detailed simulation model shows that the tetragonal prism shape of the seed has been grown from initially $19.5 \mu \mathrm{m}$ in the mean characteristic length and width direction up to approximately $60.6 \mu \mathrm{m}$ mean characteristic length and $26.55 \mu \mathrm{m}$ mean characteristic width. The length and width obtained from the detailed simulation model are very close to the target values, which are a mean characteristic length of $60.85 \mu \mathrm{m}$ and mean characteristic width of $27.36 \mu \mathrm{m}$. Based on the closed-loop operation, it can be concluded for the nominal parameter values that the controller is able to maintain the KDP concentration at its set-point for both cases while achieving the target CSD. Therefore, the next task is to evaluate the controller performance in terms of its ability to manage the uncertainties and still achieve the desired target CSD.

Here, the uncertainty and sensitivity analysis is repeated again where the same framing scenario used in the open-loop operation is applied. Thus, the same parameters from the nucleation and crystal growth rate models as shown in Table 3 as well as the same 100 samples are applied for the one- and two-dimensional case in this closed-loop analysis. The results from the Monte Carlo simulations are presented in Fig. 11 for the one-dimensional case. The KDP concentration for the one-dimensional case indicates a relatively small spread of the distribution. In comparison with openloop (Fig. 5 (left column)), it can be clearly seen that the impacts of the uncertain parameters have been minimized for the KDP concentration when operated under closed-loop conditions. The effect of the uncertain parameters is almost not visible in the temperature profiles. In addition, the variability of the final one-dimensional CSD is reduced as well, compared to the open-loop case (Fig. 5 (left column)). The significant reduction in the uncertainty in the KDP concentration and one-dimensional CSD variation compared to the open-loop simulation results in Fig. 5 (left column) is however translated into a larger variation in the inlet water temperature (the manipulated variable) as shown in Fig. 11 (bottom, left).

Similarly, the uncertainty in the KDP concentration and the CSD is reduced substantially when operating under closed-loop conditions for the two-dimensional case, as can be seen when comparing Fig. 12 (left column) to Fig. 5 (right column). Clearly, the inlet water temperature now shows a large spread due to the fact that this variable is manipulated to maintain the KDP concentration at the generated set-point. In addition, the two-dimensional case is further tested under correlation control by using the known correlation between the parameters. The two-dimensional case is chosen due to the availability of experimental data from the literature $[26,27]$. The correlation matrix calculated in this work for the twodimensional KDP crystallization is shown in Table 8. The LHS sampling was now repeated with the Iman and Conover correlation control method [22] and the resulting Monte Carlo simulations of the closed-loop system are shown in Fig. 12 (right column). Comparing results, the KDP concentration and two-dimensional CSD variation under correlation control is found to be less compared to the simulations without correlation control. This difference indicates the importance of considering correlation control in the analysis whenever information about covariance of parameters is available. Hereafter, the results of the two-dimensional case with correlation control are used for the representation of uncertainty and risk calculation.

The data are then analyzed using the representation of uncertainty using mean, 10th, and 90th percentile values of the Monte Carlo simulations. As shown in Fig. 13, the 10th and 90th
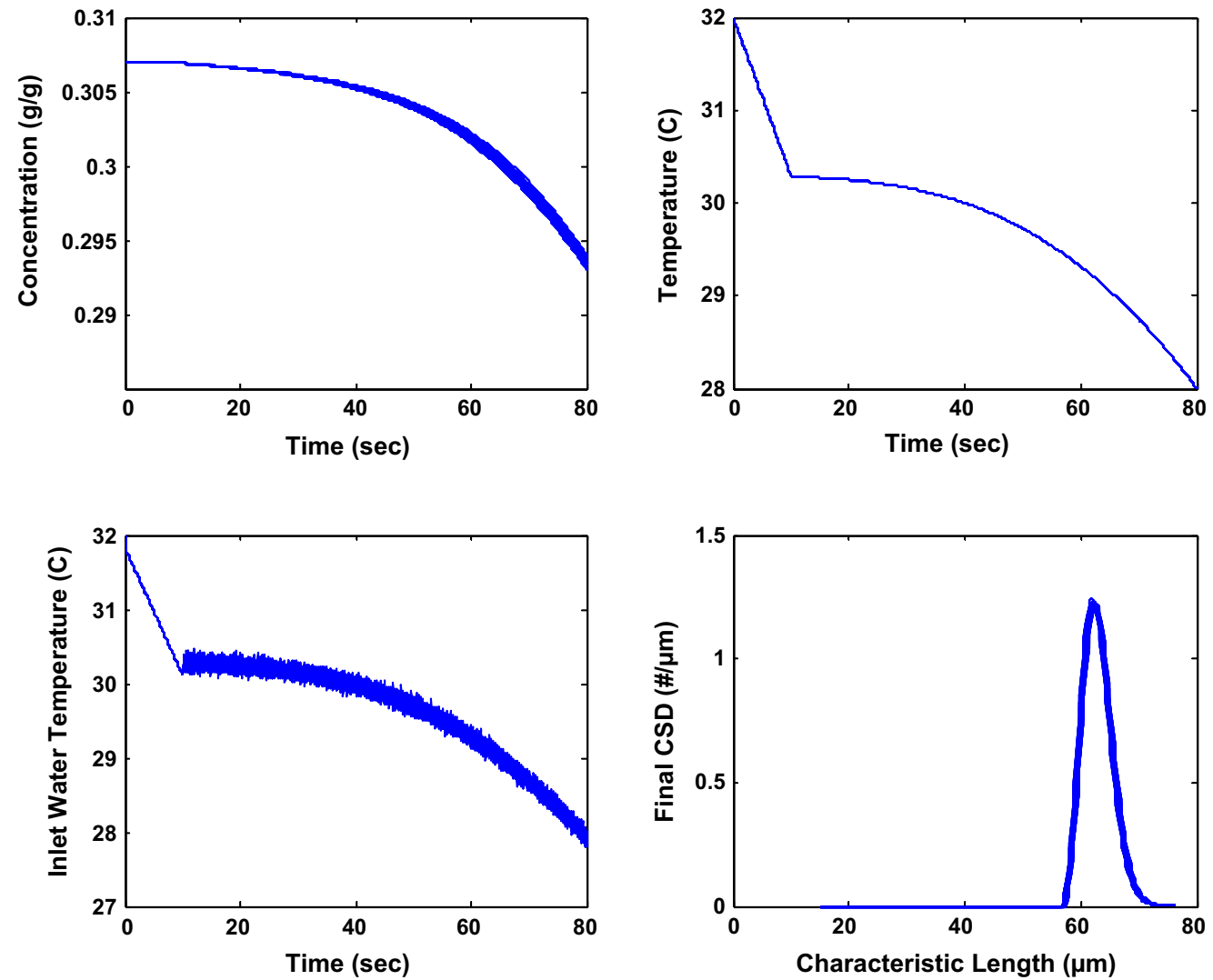

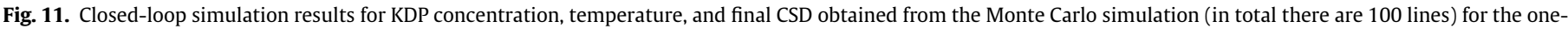
dimensional case. (For interpretation of the references to color in this figure legend, the reader is referred to the web version of this article.) 

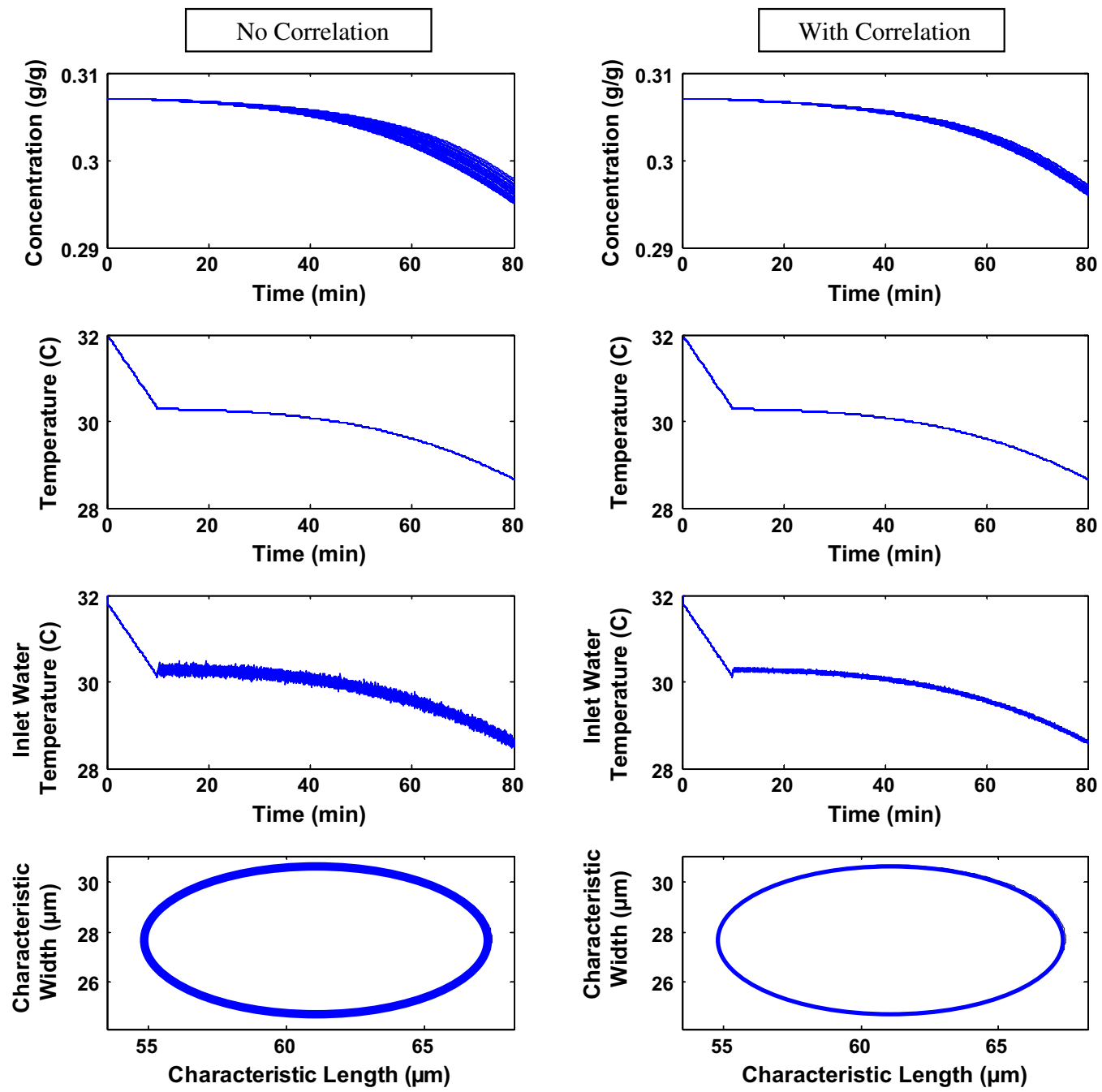

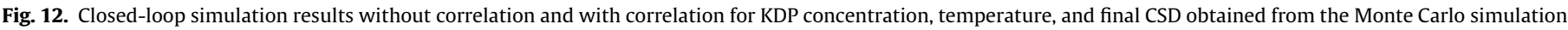

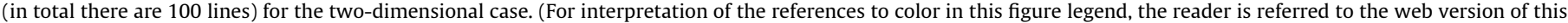
article.)

percentiles for the KDP concentration and final CSD in the oneand two-dimensional case are very close to the mean, indicating that the uncertainty is significantly reduced compared to the open-loop simulation results. The risk of not achieving the target CSD is then calculated for the one- and two-dimensional case, respectively. As mentioned earlier, the acceptable CSDs are specified in terms of mean \pm standard deviation (1.22 \pm 0.04 no. of particles $/ \mu \mathrm{m}$ for the one-dimensional case and $0.043 \pm 0.005$ no. of particles $/ \mu \mathrm{m}^{2}$ for the two-dimensional case). As shown in Fig. 14, the probability for the one- and two-dimensional cases of not achieving the target CSD is 0 , indicating that CSDs obtained for both cases are in the specified acceptable range. Thus, it can be concluded that the PAT system design using the simple PI control structure and proper controller tuning applied in this study is indeed reliable and robust enough to deal with the presence of uncertainties and is able to deliver target one- and two-dimensional CSDs. Note that the risk calculation presented here is for demonstration purposes only and is specific for the considered parametric uncertainty scenarios. The risk can also be defined in different ways to reflect the important product property attributes

Table 8

Estimated kinetic parameters with confidence interval and correlation matrix for two-dimensional KDP crystallization.

\begin{tabular}{|c|c|c|c|c|c|c|c|c|c|c|}
\hline \multirow[t]{2}{*}{ Parameters } & \multirow[t]{2}{*}{ Values } & \multirow[t]{2}{*}{ Confidence interval (95\%) } & \multicolumn{8}{|c|}{ Correlation matrix } \\
\hline & & & $k_{b}$ & $b$ & $k_{g x}$ & $g_{x}$ & $\gamma_{x}$ & $k_{g y}$ & $g_{y}$ & $\gamma_{y}$ \\
\hline$k_{b}$ & $7.49 \mathrm{E}-08$ & $\pm 3.5 \mathrm{E}-09$ & 1.000 & 0.998 & 0.914 & 0.905 & 0.886 & 0.841 & 0.812 & 0.803 \\
\hline$b$ & 2.04 & \pm 0.16 & 0.998 & 1.000 & 0.890 & 0.886 & 0.874 & 0.865 & 0.813 & 0.822 \\
\hline$k_{g x}$ & 12.21 & \pm 3.3167 & 0.914 & 0.890 & 1.000 & 0.994 & 0.987 & 0.967 & 0.903 & 0.897 \\
\hline$g_{x}$ & 1.48 & \pm 0.06 & 0.905 & 0.886 & 0.994 & 1.000 & 0.994 & 0.886 & 0.879 & 0.867 \\
\hline$\gamma_{x}$ & 0.6 & \pm 0.1 & 0.886 & 0.874 & 0.987 & 0.994 & 1.000 & 0.875 & 0.831 & 0.833 \\
\hline$k_{g y}$ & 100.75 & \pm 12.3833 & 0.841 & 0.865 & 0.967 & 0.886 & 0.875 & 1.000 & 0.989 & 0.976 \\
\hline$g_{y}$ & 1.74 & \pm 0.07 & 0.812 & 0.813 & 0.903 & 0.879 & 0.831 & 0.989 & 1.000 & 0.977 \\
\hline$\gamma_{y}$ & 0.6 & \pm 0.1 & 0.803 & 0.822 & 0.897 & 0.867 & 0.833 & 0.976 & 0.977 & 1.000 \\
\hline
\end{tabular}



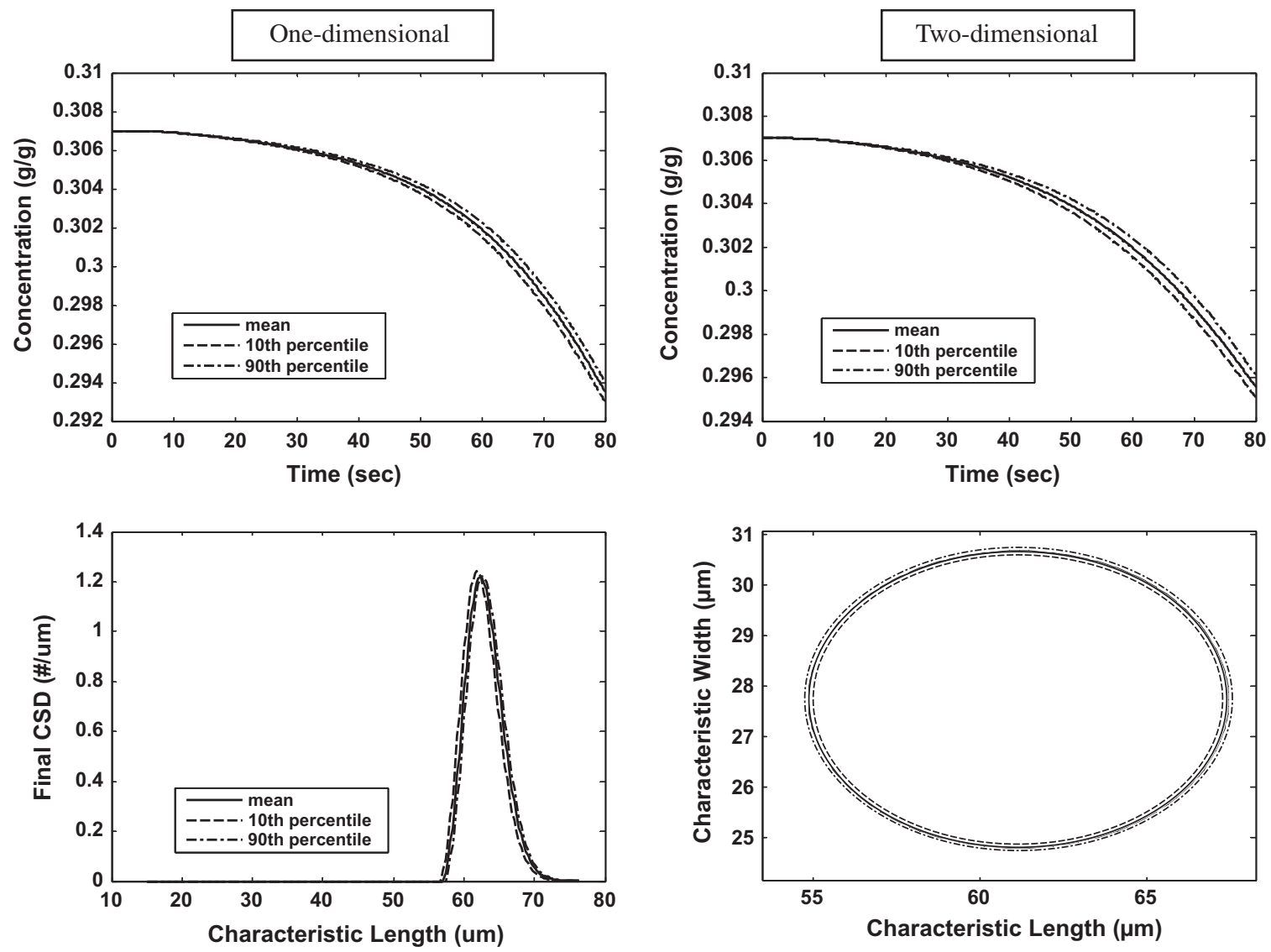

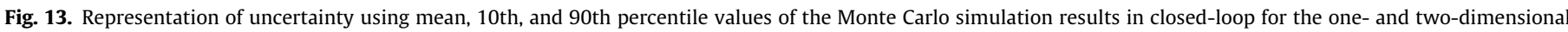
cases. Note that the reported results for the two-dimensional case include correlation control during parameter sampling.
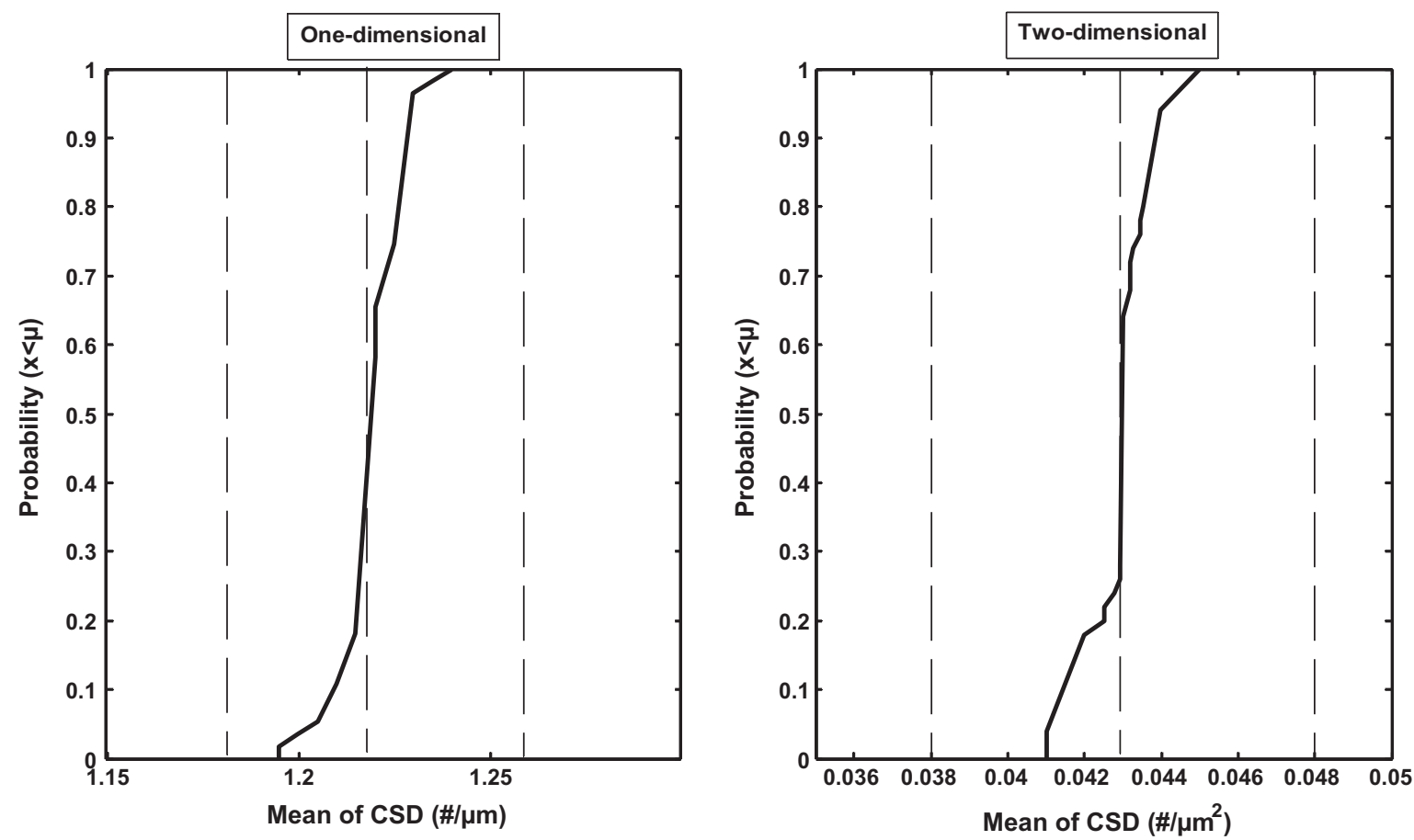

Fig. 14. Risk of not achieving the desired target for one- and two-dimensional cases in closed-loop operation.

of the pharmaceutical product, which we assume to be known by the user.
Note that in this work, the validation of the PAT system using uncertainty and sensitivity analysis is carried out under 
open- and closed-loop conditions. The simulation under open-loop conditions uses a natural cooling profile, and the closed-loop simulation is based on a cubic cooling profile. This is in line with our objective of using the open-loop simulations with natural cooling profile to complement the study of understanding the effect of parametric uncertainties on the model prediction. Clearly, the parametric uncertainties affect the model predictions significantly in open-loop, and these effects are then studied with closed-loop simulations to verify the robustness of the proposed PAT system which uses a feedback controller.

The comparison of the effect of parametric uncertainties in open- and closed-loop using the same cooling strategy has also been performed, and the results are given in Appendix $C$ as Supplementary information. In this case, the effect of secondary nucleation is eliminated in open-loop (no secondary peak on CSDs observed for both cases). As can be noted from Appendix C, the spread of the CSD variation obtained under open-loop simulation conditions is in that case a little higher than the CSD obtained under closed-loop simulation conditions for both the one- and the two-dimensional cases. This indicates that the reduction of uncertainty in the KDP concentration and CSDs is the result of a combination of the selected temperature cooling trajectory as well as the action of the feedback controller itself. That said the CSDs obtained under closed-loop simulation conditions have a lower variance $\left(\sigma^{2}=6.4 \times 10^{-5}\right.$ for the one-dimensional case and $\sigma^{2}=2.56 \times 10^{-6}$ for the two-dimensional case) compared to the CSDs obtained for the open-loop conditions $\left(\sigma^{2}=1.44 \times 10^{-4}\right.$ for the one-dimensional case and $\sigma^{2}=1.3 \times 10^{-5}$ for the two-dimensional case), still indicating the need for and the effectiveness of the feedback controller to enhance the robustness against uncertainties in crystallization kinetics.

\section{Step 5.2: Decision making}

Based on the validation of the PAT system under closed-loop operation using uncertainty and sensitivity analysis, it is concluded that the designed PAT system used in this study is able to achieve the target CSDs under the considered domain of uncertainties. There is a clear improvement of the probability of not achieving the target CSD calculated in the open-loop scenario (probability of 0.75 and 0.8 for one- and two-dimensional cases, respectively) and the probability calculated in the closed-loop scenario (probability of 0 for both cases). Therefore, this PAT system design is now ready to be implemented in the next step. However, it is also important to point out that there are options in this developed framework for PAT system design in the case of a probability of not achieving the target CSD is obtained which is exceeding the specified probability of 0.05 (assumed to be a minimum requirement, see above). Potential options for reducing the probability are by improving the controller in terms of re-tuning the PID controller, or using more advanced control, or as a final option to investigate whether the target CSD requirements cannot be relaxed without causing problems for the subsequent processing steps of the crystal product.

\subsection{Implementation of the designed PAT system (Step 6)}

Based on the outcome of Steps 4 and 5, a proposal for a PAT system has been obtained and is ready to be implemented. An example of a PAT system implementation in a simulation model has been illustrated in our earlier work [1] and is therefore not shown here. Note that the designed PAT system in our earlier work assumes that there are no uncertainties in the kinetic parameters. Here, the PAT system performance has been comprehensively verified using uncertainty and sensitivity analysis considering parametric uncertainty of crystallization kinetics, which further demonstrate the reliability of the PAT system under the considered domain of uncertainties.

\section{Future perspectives}

In this work, only kinetic parameters in the nucleation and crystal growth rate expressions were considered as an input uncertainty. There is also uncertainty present in the initial conditions, for example the seed crystals. Seeding is an efficient approach to stabilize the crystallization process and produce the desired target product. Usually, the amount and size of seeds to be added to the crystallizer is determined from experimental data which contribute to some extent to the uncertainties. Therefore also, it could be interesting in the future to consider the properties of the seed crystals as an input uncertainty as well. As indicated earlier, implementation of the proposed PAT system in a real crystallization process could both be used to verify and confirm the methodology and the results obtained here.

\section{Conclusions}

The methodology for implementing uncertainty and sensitivity analysis has been successfully implemented in the model-based systematic design framework for monitoring and control (PAT) systems of crystallization processes. The application of uncertainty and sensitivity analysis has been highlighted through a case study using a one- and a two-dimensional KDP crystallization process model. These models have been applied in the frame of the model-based design of a PAT system, for both open-loop and closedloop scenarios. In this work, we focused on the crystallization kinetics as source of uncertainties, namely the parameters of the nucleation and the crystal growth rate were propagated using the Monte Carlo procedure. In open-loop using natural cooling profiles, as expected, Monte Carlo simulation results in a large spread of the simulated KDP concentration and the CSD obtained for both cases, and accordingly, the risk of not achieving the target CSD is high. Subsequently, global sensitivity analysis has been performed using the SRC method. Results were verified with the Morris screening method, which indicated that the most significant parameters affecting output variance are nucleation order, $b$ (secondary peak) and growth order constants, $g_{x}$ (seed of the CSD). A significant reduction of variation in the KDP concentration and the CSDs was obtained in closed-loop for the one-dimensional as well as the two-dimensional KDP model. For the two-dimensional KDP case study, it is also demonstrated that including the covariance of uncertain parameters in the Monte Carlo uncertainty analysis influences the calculated output variance of the CSD (hence the associated decision making based on the results). This indicates that covariance information between sources of uncertainties should be included in the analysis whenever such information is available. Based on the simulation results, the uncertainty on the final CSD could be characterized by an empirical cumulative distribution function (ECDF) from which the probability of not achieving the target CSDs (or a specified range of target CSD) is obtained which essentially provides the risk associated with a certain PAT design. For the case studies presented, the risk of not achieving the target CSD for the proposed PAT system design was found to be zero, thus verifying the robustness of the PAT system design under the considered parametric uncertainty scenarios.

\section{Acknowledgements}

The financial support for this PhD project provided by the Malaysian Ministry of Higher Education (MoHE) and Universiti Malaysia Pahang (UMP) is gratefully acknowledged. 
Table A.1

List of model equations for the two-dimensional model of KDP crystallization.

\begin{tabular}{|c|c|c|}
\hline No. & Type of equations & Equations \\
\hline 1. & Population balance equation & $\frac{d N_{i, j}}{d t}+f_{i, j}+f_{i, j}^{\prime}=B_{n u c}, i=1, \ldots, n ; j=1, \ldots, m$ \\
\hline 2. & Overall mass balance: solute concentration & $\frac{d c}{d t}=-\frac{\rho_{c}}{m_{w}}\left(\sum_{i, j}\left(\frac{1}{3} S_{y j}^{3}\left(S_{x i}-S_{y j}\right) S_{y j}^{2}\right) \frac{d N_{i, j}}{d t}\right)$ \\
\hline 3. & Energy balance & $\rho V c_{p} \frac{d T}{d t}=-\Delta H_{c} \rho_{c} V\left(\sum_{i, j}\left(\frac{1}{3} S_{y j}^{3}\left(S_{x i}-S_{y j}\right) S_{y j}^{2}\right) \frac{d N_{i, j}}{d t}\right)-U_{1} A_{1}\left(T-T_{w}\right)$ \\
\hline 4. & Cooling jacket energy balance & $\rho_{w} V_{w} c_{p w} \frac{d T_{w}}{d t}=\rho_{w} F_{w i n} c_{p w}\left(T_{w i n}-T_{w}\right)+U_{1} A_{1}\left(T-T_{w}\right)+U_{2} A_{2}\left(T_{e x}-T_{w}\right)$ \\
\hline 5. & Saturation concentration & $c^{\text {sat }}=a_{i 1}+b_{i 1} T+c_{i 1} T^{2}+d_{i 1} T^{3}$ \\
\hline 6. & Supersaturation & $S=\frac{c--^{\text {sat }}}{c^{\text {sat }}}$ \\
\hline 7. & Nucleation & $B_{\text {nuc }}=k_{b} S^{b} V$ \\
\hline 8. & Crystal growth rate (length direction) & $G_{x i}=0.1 k_{g x} S^{g_{x}}\left(1+\gamma_{x} L_{x i}\right)^{p_{x}}$ \\
\hline 9. & Crystal growth rate (width direction) & $G_{y j}=0.1 k_{g y} S^{g_{y}}\left(1+\gamma_{y} L_{y j}\right)^{p_{y}}$ \\
\hline 10. & Tailor development coefficient for length classes & $a_{x i}=\frac{\Delta C l_{i+1}}{\Delta C l_{i}\left(\Delta C l_{i+1}+\Delta C l_{i}\right)}$ \\
\hline 11. & Tailor development coefficient for length classes & $b_{x i}=\frac{\Delta C l_{i}}{\Delta C l_{i+1}\left(\Delta C l_{i+1}+\Delta C C_{i}\right)}$ \\
\hline 12. & Tailor development coefficient for width classes & $c_{y j}=\frac{\Delta C l_{j+1}}{\Delta C C_{j}\left(\Delta C l_{j+1}+\Delta C l_{j}\right)}$ \\
\hline 13. & Tailor development coefficient for width classes & $d_{y j}=\frac{\Delta C l_{j}}{\Delta C l_{j+1}\left(\Delta C l_{j+1}+\Delta C l_{j}\right)}$ \\
\hline 14. & Characteristic length & $S_{x i}=\frac{L_{x i}-L_{x i-1}}{2}$ \\
\hline 15. & Characteristic width & $S_{y j}=\frac{L_{y j}-L_{y j-1}}{2}$ \\
\hline 16. & Outlet crystal flux for length direction & $f_{i, j}^{1, O}=G_{x i}\left(a_{x i} N_{i, j}+b_{x i} N_{i+1, j}\right)$ \\
\hline 17. & Inlet crystal flux for length direction & $f_{i, j}^{1, I}=G_{x i}\left(a_{x i-1} N_{i-1, j}+b_{x i-1} N_{i, j}\right)$ \\
\hline 18. & Outlet crystal flux for width direction & $f_{i, j}^{2,0}=G_{y j}\left(c_{y j} N_{i, j}+d_{y j} N_{i, j+1}\right)$ \\
\hline 19. & Inlet crystal flux for width direction & $f_{i, j}^{2, I}=G_{y j}\left(c_{y j-1} N_{i, j-1}+d_{y j-1} N_{i, j}\right)$ \\
\hline 20. & Inlet flow for length direction & $f_{i, j}=f_{i, j}^{1, O}-f_{i, j}^{1, I}$ \\
\hline 21. & Inlet flow for width direction & $f_{i, j}^{\prime}=f_{i, j}^{2,0}-f_{i, j}^{2, I}$ \\
\hline 22. & Total number of particles & $N_{c}=\sum_{i, j} N_{i, j}$ \\
\hline 23. & Total crystal mass & $M_{c}=\rho_{c} \sum_{i, j}\left(\frac{1}{3} S_{y j}^{3}\left(S_{x i}-S_{y j}\right) S_{y j}^{2}\right) N_{i, j}$ \\
\hline 24. & Crystal size distribution & $f_{n}\left(L_{x i}, L_{y j}\right)=\frac{\frac{N_{i, j}}{\Delta C_{i} \Delta C_{j}}+\frac{N_{i+1, j+1}}{\Delta C_{i+1} \Delta C_{j+1}}}{2}$ \\
\hline
\end{tabular}

\section{Appendix A.}

Table A.1.

Table B.1

Method comparison for screening influential factors based on the two-dimensional CSD data taken at p1 in Fig. 9.

\begin{tabular}{lllllr}
\hline \multirow{2}{*}{ Ranking } & \multicolumn{2}{l}{ Morris screening method } & & \multicolumn{2}{l}{ SRC method } \\
\cline { 2 - 3 } \cline { 5 - 6 } & Parameters & $\mu_{j}$ & & Parameters & \multicolumn{1}{l}{$\beta_{j k}$} \\
\hline 1 & $b$ & -0.8563 & & $b$ & -0.9032 \\
2 & $g_{x}$ & 0.2561 & & $g_{x}$ & 0.2312 \\
3 & $g_{y}$ & 0.2043 & & $g_{y}$ & 0.1932 \\
4 & $\gamma_{x}$ & -0.1201 & & $\gamma_{x}$ & -0.1671 \\
5 & $\gamma_{y}$ & -0.1143 & & $\gamma_{y}$ & -0.0921 \\
6 & $k_{b}$ & 0.0495 & & $k_{b}$ & 0.0851 \\
7 & $k_{g x}$ & -0.0392 & & $k_{g x}$ & -0.0212 \\
8 & $k_{g y}$ & -0.0065 & & $k_{g y}$ & -0.0128 \\
\hline
\end{tabular}

Table B.2

Method comparison for screening influential factors based on the two-dimensional CSD data taken at 22 in Fig. 9.

\begin{tabular}{lllllr}
\hline \multirow{2}{*}{ Ranking } & \multicolumn{2}{l}{ Morris sampling method } & & \multicolumn{2}{l}{ SRC method } \\
\cline { 2 - 3 } & Parameters & $\mu_{j}$ & & Parameters & $\beta_{j k}$ \\
\hline 1 & $g_{x}$ & 0.5921 & & $g_{x}$ & 0.6641 \\
2 & $g_{y}$ & 0.5843 & & $g_{y}$ & 0.5395 \\
3 & $\gamma_{x}$ & -0.3094 & & $\gamma_{x}$ & -0.3781 \\
4 & $\gamma_{y}$ & -0.2895 & & $\gamma_{y}$ & -0.3021 \\
5 & $k_{g x}$ & 0.1153 & & $k_{g x}$ & 0.1643 \\
6 & $k_{g y}$ & 0.0843 & & $k_{g y}$ & 0.1296 \\
7 & $b$ & -0.0201 & & $b$ & -0.0403 \\
8 & $k_{b}$ & -0.0184 & & $k_{b}$ & -0.0276 \\
\hline
\end{tabular}

\section{Appendix B.}

Tables B.1 and B.2.

\section{Appendix C. Supplementary material}

Supplementary data associated with this article can be found, in the online version, at http://dx.doi.org/10.1016/j.ejpb.2013.05.016.

\section{References}

[1] N.A.F.A Samad, G. Sin, K.V. Gernaey, R. Gani, A systematic framework for design of process monitoring and control (PAT) systems for crystallization processes, Comput. Chem. Eng. 54 (2013) 8-23.

[2] R. Singh, K.V. Gernaey, R. Gani, Model-based computer-aided framework for design of process monitoring and analysis systems, Comput. Chem. Eng. 33 (2009) 22-42.

[3] Z.K. Nagy, R.D. Braatz, Advances and new directions in crystallization control, Annu. Rev. Chem. Biomol. Eng. 3 (2012) 55-75.

[4] A. Saengchan, P. Kittisupakorn, W. Paengjuntuek, A. Arpornwichanop, Improvement of batch crystallization control under uncertain kinetic parameters by model predictive control, J. Ind. Eng. Chem. 17 (2011) 430-438

[5] Z.K. Nagy, Model based robust control approach for batch crystallization product design, Comput. Chem. Eng. 33 (2009) 1685-1691.

[6] Z.K. Nagy, R.D. Braatz, Robust nonlinear model predictive control of batch processes, AIChE J. 49 (7) (2003) 1776-1786.

[7] Z.K. Nagy, R.D. Braatz, Open-loop and closed-loop robust optimal control of batch processes using distributional and worst-case analysis, J. Process Contr. 14 (2004) 411-422.

[8] D.L. Ma, S.H. Chung, R.D. Braatz, Worst-case performance analysis of optimal batch control trajectories, AIChE J. 45 (7) (1999) 1469-1476.

[9] G. Sin, K.V. Gernaey, M.B. Neumann, M.C.M. van Loosdrecht, W. Gujer, Uncertainty analysis in WWTP model applications: a critical discussion using an example from design, Water Res. 43 (2009) 2894-2906.

[10] M. Omlin, P. Reichert, A comparison of techniques for the estimation of mode prediction uncertainty, Ecol. Model. 115 (1999) 45-59.

[11] X. Flores-Alsina, I. Rodriguez-Roda, G. Sin, K.V. Gernaey, Uncertainty and sensitivity analysis of control strategies using the benchmark simulation model No1 (BSM1), Water Sci. Technol. 59 (3) (2009) 491-499. 
[12] J.C. Helton, F.J. Davis, Latin hypercube sampling and the propagation of uncertainty in analyses of complex systems, Reliab. Eng. Syst. Safe. 81 (2003) 23-69.

[13] F.E. Haskin, B.D. Staple, C. Ding, Efficient uncertainty analyses using fast probability integration, Nucl. Eng. Des. 166 (1996) 225-248.

[14] A. Saltelli, M. Ratto, S. Tarantola, F. Campolongo, Sensitivity analysis practices: strategies for model-based inference, Reliab. Eng. Syst. Safe. 91 (2006) 11091125.

[15] A. Saltelli, M. Ratto, T. Andres, F. Campolongo, J. Cariboni, D. Gatelli, M. Saisana, S. Tarantola, Global Sensitivity Analysis: The Primer, John Wiley \& Sons, West Sussex, England, 2008.

[16] G. Sin, K.V. Gernaey, M.B. Neumann, M.C.M. van Loosdrecht, W. Gujer, Global sensitivity analysis in wastewater treatment plant model applications: prioritizing sources of uncertainty, Water Res. 45 (2011) 639-651.

[17] M.D. Morris, Factorial sampling plans for preliminary computational experiments, Technometrics 33 (1991) 161-174.

[18] J. Cariboni, D. Gatelli, R. Liska, A. Saltelli, The role of sensitivity analysis in ecological modelling, Ecol. Model. 203 (2007) 167-182.

[19] F. Campolongo, J. Cariboni, A. Saltelli, An effective screening design for sensitivity analysis of large models, Environ. Modell. Softw. 22 (2007) 15091518.

[20] M.S. Grewal, A.P. Andrews, Kalman Filtering: Theory and Practice using MATLAB, third ed., John Wiley \& Sons, New Jersey, 2008.

[21] A. Mesbah, A.E.M. Huesman, H.J.M. Kramer, P.M.J. Van den Hof, A comparison of nonlinear observers for output feedback model-based control of seeded batch crystallization processes, J. Process. Contr. 21 (2011) 652-666.

[22] R.L. Iman, W.J. Conover, A distribution-free approach to inducing rank correlation among input variables, Commun. Stat. - Simul. C 11 (3) (1982) 311-334.

[23] F. Campolongo, A. Saltelli, Sensitivity analysis of an environmental model: an application of different analysis methods, Reliab. Eng. Syst. Safe. 57 (1997) 4969.

[24] N.A.F.A. Samad, R. Singh, G. Sin, K.V. Gernaey, R. Gani, A generic multidimensional model-based system for batch cooling crystallization processes, Comput. Chem. Eng. 35 (2011) 828-843.

[25] M. Heitzig, G. Sin, M. Sales-Cruz, P. Glarborg, R. Gani, Computer-aided modeling framework for efficient model development, analysis and identification: combustion and reactor modeling, Ind. Eng. Chem. Res. 50 (2011) 5253-5265.

[26] R. Gunawan, D.L. Ma, M. Fujiwara, R.D. Braatz, Identification of kinetic parameters in multidimensional crystallization processes, Int. J. Mod. Phys. B 16 (2002) 367-374.

[27] R. Gunawan, I. Fusman, R.D. Braatz, High resolution algorithms for multidimensional population balance equations, AIChE J. 50 (11) (2004) 2738-2749.

[28] K. Lee, J.H. Lee, M. Fujiwara, D.L. Ma, R.D. Braatz, Run-to-run control of multidimensional crystal size distribution in a batch crystallizer, Proc. Am. Contr. Conf. 1-6 (2002) 1013-1018. 